\title{
Neurobehavioral toxicology of pyrethroid insecticides in adult animals: A critical review ${ }^{2}$
}

\author{
M.J. Wolansky ${ }^{\mathrm{a}, \mathrm{b}, *}$, J.A. Harrill ${ }^{\mathrm{a}, \mathrm{c}}$ \\ ${ }^{a}$ Neurotoxicology Division, National Health and Environmental Effects Research Laboratory, Office of Research and Development, USEPA, RTP, NC, United States \\ ${ }^{\mathrm{b}}$ U.S. National Research Council, United States \\ ${ }^{\mathrm{c}}$ Curriculum in Toxicology, University of North Carolina, Chapel Hill, NC, United States
}

Received 13 July 2007; received in revised form 22 October 2007; accepted 28 October 2007

\begin{abstract}
Pyrethroids are pesticides with high selectivity for insects. In order to identify strengths and gaps in the database for pyrethroid neurobehavioral toxicology, we have critically analyzed the data from peer-reviewed literature. This review includes dose-response data that have been recently generated demonstrating consistent findings for low-dose, acute, oral exposure to pyrethroids in small rodents. All pyrethroids tested (i.e., about twenty compounds), regardless of structure, produce a decrease in motor activity in a variety of test protocols. The range of relative potencies varies more than two orders of magnitude, and thresholds for motor activity were found well below doses that produce overt signs of poisoning. Six compounds (allethrin, permethrin, cis-permethrin, deltamethrin, cypermethrin, and fenvalerate) impair schedule-controlled operant responding, seven compounds (pyrethrum, bifenthrin, $S$-bioallethrin, permethrin, $\beta$-cyfluthrin, cypermethrin, and deltamethrin) decrease grip strength, and two compounds (deltamethrin and $\alpha$ cypermethrin) produce incoordination using the rotarod. In addition, while compounds lacking an $\alpha$-cyano group (e.g., cismethrin, permethrin, bifenthrin) induce an increase in acoustic-evoked startle response amplitude, cyano compounds (e.g., deltamethrin, cypermethrin, cyfluthrin) produce the opposite outcome. Other endpoints (e.g., tremor intensity, sensory response) have been only occasionally explored. A synthesis of the neurobehavioral evidence relating to the action of pyrethroids indicates that some differences in the experimental findings across compounds are also present in the low-effective dose range. For risk assessment purposes, a strategy that takes into account data from an array of neurobehavioral endpoints is needed to capture the heterogeneity of pyrethroid-induced adverse effects and accurately inform policy decisions.
\end{abstract}

Published by Elsevier Inc.

Keywords: Pyrethroids; Neurobehavioral toxicology; Mammals; Risk assessment

\section{Contents}

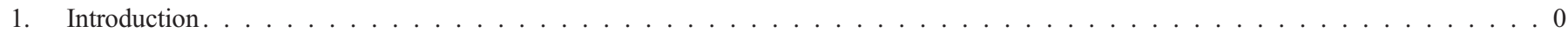

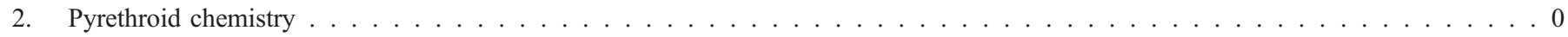

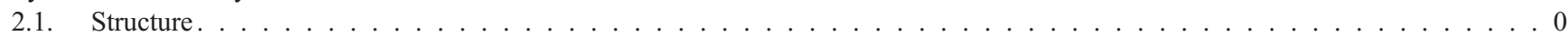



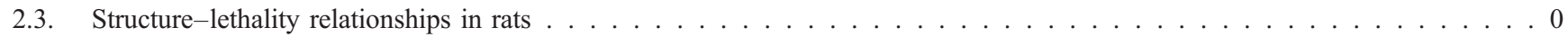

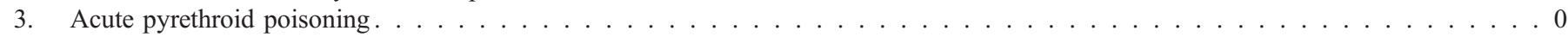

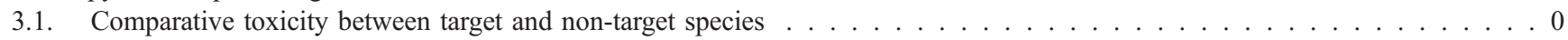

3.2. Signs of pyrethroid poisoning in rodents and classification schemes . . . . . . . . . . . . . . . . . . . . . . 0

\footnotetext{
t5 This manuscript has been reviewed by the National Health and Environmental Effects Research Laboratory, U.S. Environmental Protection Agency, and approved for publication, but does not reflect USEPA policy. Any mention of trade names or commercial products does not constitute endorsement or recommendation for use.

* Corresponding author. Present address: Facultad de Ciencias Exactas y Naturales (Universidad de Buenos Aires). Int. Guiraldes 2160, Pabellón 2, Piso 4, Ciudad Universitaria, Buenos Aires (C1428EGA), Argentina. Tel.: +54 1148547576.

E-mail address: mjwolansky@gmail.com (M.J. Wolansky).
} 


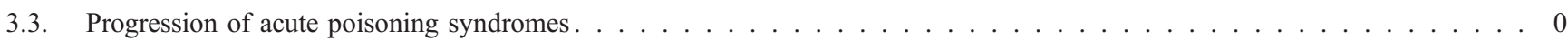

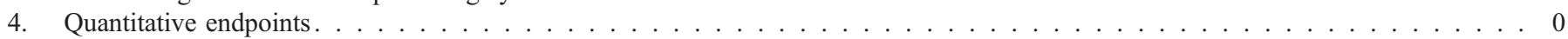

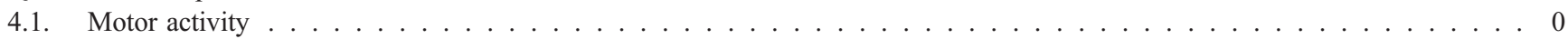

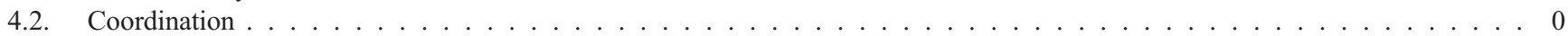

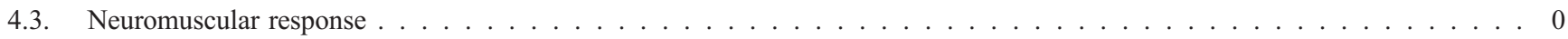

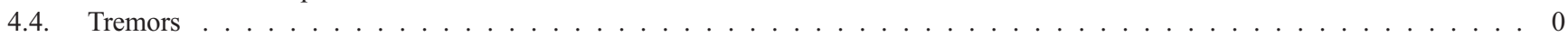

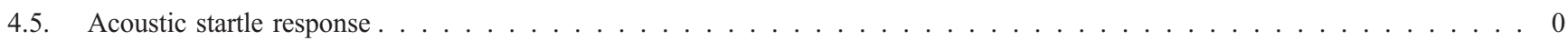

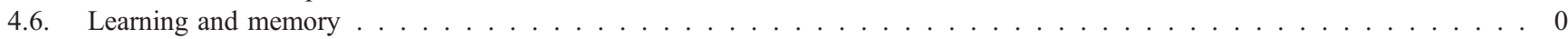

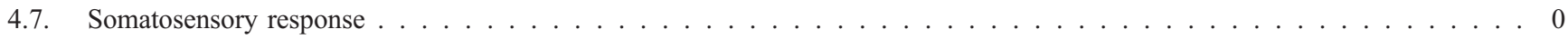

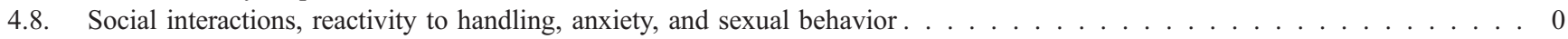

4.9. Other descriptions of pyrethroid-induced neurobehavioral toxicity. . . . . . . . . . . . . . . . . . . . . . . 0

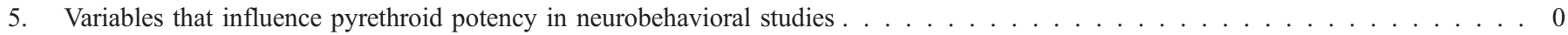

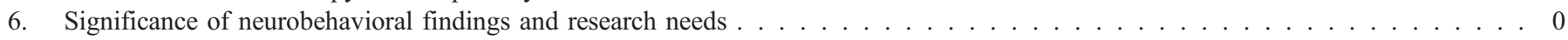

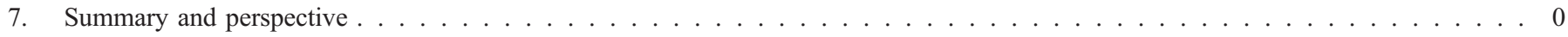

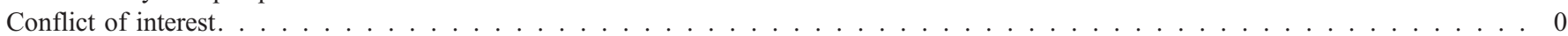

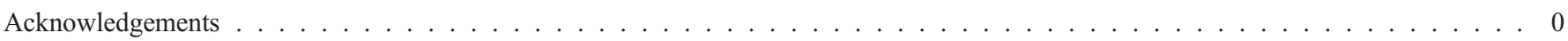

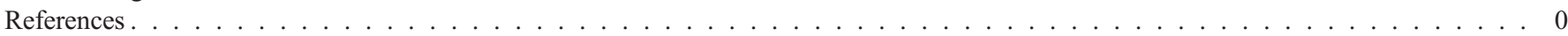

\section{Introduction}

Pyrethroids are a class of neurotoxic pesticides registered for agricultural and residential use in the United States. Use of pyrethroids has continuously increased during the last two decades [21,19,40,58]. Evaluations of tolerances for individual pyrethroid applications, as well as the potential for cumulative risk of neurotoxicity following exposure to multiple pyrethroids, are currently underway in accordance with the mandate of the Food Quality Protection Act [56]. The impending regulatory decisions will be based in part on laboratory studies of the neurotoxic effects of pyrethroids conducted in both in vitro and in vivo models. One area of research that will potentially inform these risk decisions are observations of behavior in laboratory rodents.

A large database of behavioral observations recorded following pyrethroid exposure is available in the peer-reviewed literature and reports from regulatory agencies. Some of these studies describe signs of excessive toxicity observed following lethal or near-lethal pyrethroid exposures while other studies describe behavioral effects observed at lower dose ranges which are more relevant to the risk assessment process. The primary objectives of this article are: 1) to outline the signs and progression of pyrethroid poisoning in non-target mammalian species; 2) to review the accumulated data for pyrethroid-mediated effects on rodent behavior available in the peer-reviewed literature; 3 ) to comment on the consistencies and inconsistencies of the observed neurobehavioral effects across both compounds and studies; and 4) to evaluate the utility of the reviewed evidence in determining neurotoxic risk to humans. Unfortunately, there is a lack of peerreviewed neurobehavioral studies using subchronic or chronic pyrethroid exposures which may more accurately reflect real-life exposure scenarios in human populations. Therefore, particular emphasis is placed on studies that measure quantifiable outcomes in adult rats and mice following acute, sublethal, oral exposures, as these studies comprise a majority of the data available for guiding risk decisions. Pyrethroids are metabolized rapidly in mammals [156], and no cumulative effects are observed in rats following repeated oral dosing of pyrethroids using moderately effective doses once a day for 30 days [33]. This strongly suggests that data from acute exposure studies may accurately represent the adverse outcomes observed following low-dose, chronic or subchronic exposures. Neurobehavioral effects in animals exposed to pyrethroids during development will not be included in this article, as this information has been recently reviewed elsewhere [154].

Even though a database for pyrethroid-mediated effects on rodent behavior is available, comparison across studies is complicated by several factors that may influence the observed outcomes. These factors include differences in species, strain, gender, and body weight of the animals; route of administration; vehicle and test period; pyrethroid compound purity and isomer composition [27,36,47,101,117,156,178,189,195]. A critical analysis of the experimental or biological factors that influence pyrethroid potency is not presented in this review, save for cases in which disparate results for comparable neurobehavioral endpoints are observed across studies. For reviews of the molecular actions of pyrethroids on voltage-sensitive ion channels (the primary molecular targets of pyrethroids), the reader is referred to Narahashi et al. [123], Narahashi [122], Soderlund et al. [156], and Shafer and Meyer [153].

The present article includes a brief synopsis of pyrethroid chemistry and stereochemistry to orient the reader to this pesticide class, detailed descriptions of acute pyrethroid poisoning in mammals, and a compendium of studies that assess pyrethroid effects on different domains of mammalian behavior. Finally, a discussion regarding the current body of neurobehavioral data in guiding the pyrethroid risk assessment process is provided.

\section{Pyrethroid chemistry}

\subsection{Structure}

Pyrethroids are structural derivatives of naturally occurring pyrethrins, which are present in pyrethrum, an extract from the flowers Chrysanthemum cinerarifolium [59,20,21]. Most pyrethroids contain cyclopropane carboxylic acid moieties (or an 
equivalent group) linked to aromatic alcohols through a central ester (or ether) bond (Fig. 1). Modifications to this basic pyrethroid structure are designed to increase insecticidal potency or photostability but may also result in changes in pyrethroid activity in non-target species $[60,73,193,172,177,126,156]$. One such modification is the addition of an $\alpha$-cyano group to the alcohol moiety, considered a milestone in the development of synthetic pyrethrin analogs due to its critical role in providing superior insecticidal activity $[48,61,194,156]$. Likewise, this chemical group confers an increase in potency of approximately one order of magnitude in studies of acute lethality in rodents [98,173,172,156,184].

In addition to the $\alpha$-cyano group, other modifications to the fundamental acid and alcohol moieties of pyrethroids have been introduced: namely, variations in the identity and position of halogenated and hydrophobic chemical groups and the stereochemical arrangement of these groups [126]. In the rat, these modifications account for variations in potency of almost three
Type I (non-cyano)
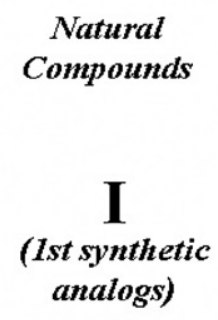

II

(aromatic alcohols)<smiles>C=C/C=C\CC1=C(C)C(OC(=O)C2C(C=C(C)C)C2(C)C)CC1=O</smiles>
pyrethrin I<smiles>C=CCC1=C(C)C(OC(=O)C2C(C=C(C)C)C2(C)C)CC1=O</smiles>

allethrin

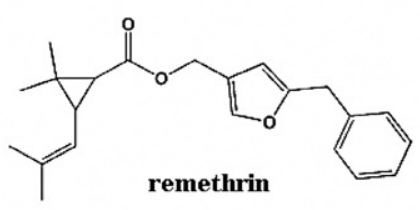

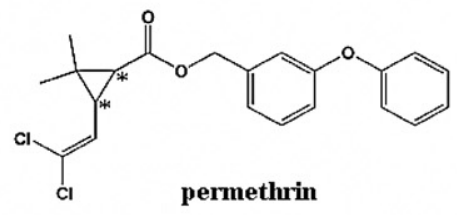

permethrin

\section{Type II ( $\alpha$-cyano)}

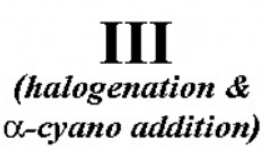

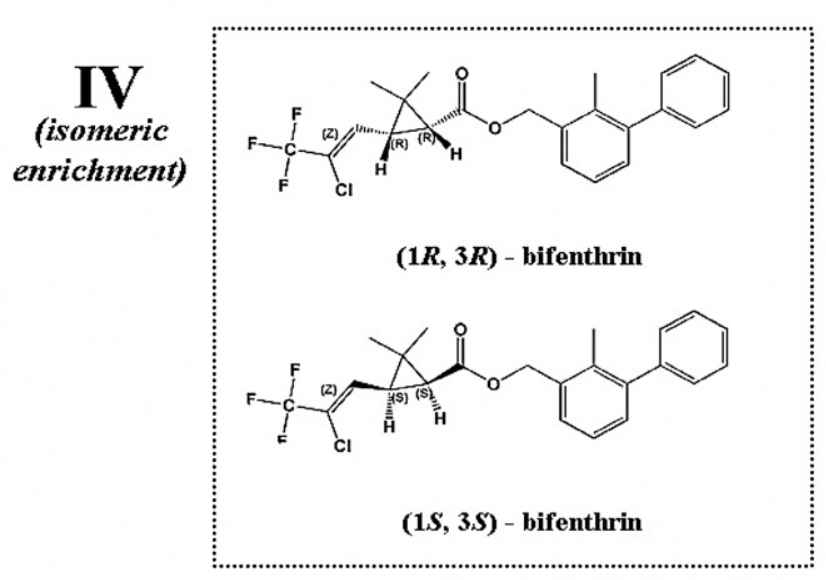

bifenthrin

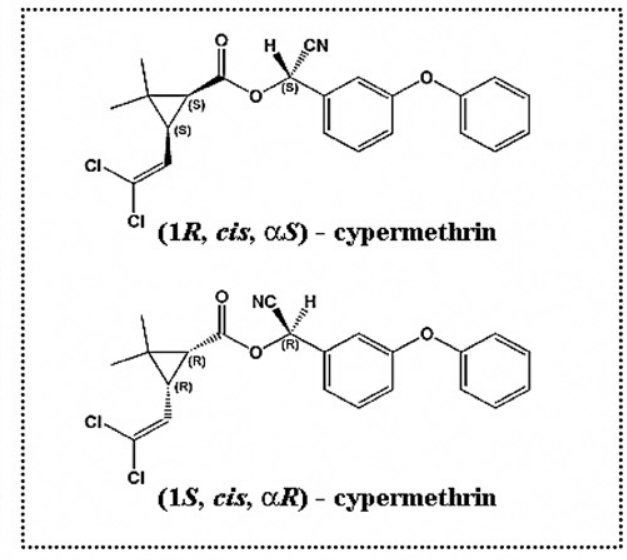

$\alpha$-cypermethrin

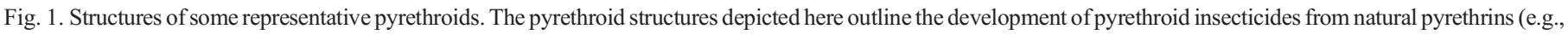

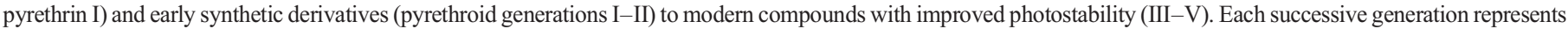

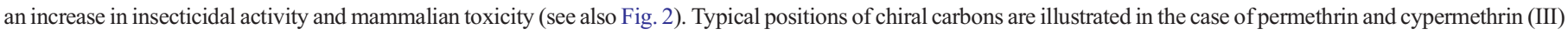

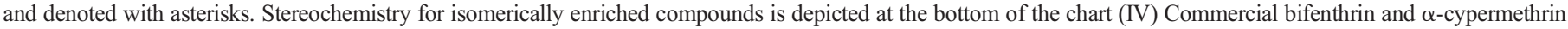

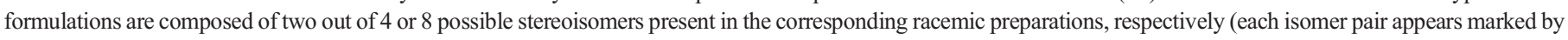
squares). The presence of an $\alpha$-cyano group on the alcohol confers increased potency in target and non-target species. 
orders of magnitude, ranging from tefluthrin, with an oral LD50 of $22 \mathrm{mg} / \mathrm{kg}$, to etofenprox, with oral LD50>10,000 mg/kg [184]. For example, bifenthrin differs little from permethrin in structure and some of the physical properties of the two (e.g., octanol-water partitioning coefficient) are similar as well $[147,97]$. Yet, bifenthrin has a $10-$ to 100 -fold lower oral LD50 in rats as compared to permethrin [112,184,156]. Computational structure-activity relationship studies indicate that the central portion of a pyrethroid molecule (i.e. components of the central ester bond) is essential for toxicity $[125,55,129]$.

\subsection{Stereochemistry}

Pyrethroids are chiral molecules and the spatial conformation of a pyrethroid compound is a major determinant of biological activity [66]. Chirality also has an impact on the biological activity of other pesticide classes [96,102]. Pyrethroids may have $2-4$ chiral centers (Fig. 1). Stereoisomers of the same compound display a wide range of potencies in both target and non-target species $[175,66,28,96,102]$. For most pyrethroids, preparations enriched in cis-isomers (e.g., cisresmethrin, cis-permethrin, cis-cypermethrin, and cis-isomers of cyhalothrin) have greater potency (i.e., lower oral LD50) in rodents than corresponding preparations with isomeric heterogeneity $[182,112,135,184]$. For instance, cismethrin, the $1 R$-cisisomer of resmethrin, is at least 50 -fold more potent (i.e., has a 50 -times lower LD50) than the $1 R$-trans-isomer in rats, regardless of the route of exposure $[1,180,176,75,30]$, and $\sim 30$-fold more potent than resmethrin in decreasing motor activity $[33,187]$.

Pyrethroids with an $\alpha$-cyano group can also assume different isomeric configurations based on the spatial orientation of this group. The $[\mathrm{S}]$ configuration confers higher activity than the $[R]$ configuration in target and non-target species $[48,172,156]$. A comparison of the toxicity of cypermethrin and $\alpha$-cypermethrin preparations in rodents exemplifies the increased activity in compounds enriched in cis-isomers that include an $\alpha S$-cyano group. Both cypermethrin preparations are composed of $50 \%$ cisisomers. The racemic mixture of cypermethrin stereoisomers has an isomer ratio of 2:2:2:2, $\alpha R$-cyano (1RS)-cis, $\alpha R$-cyano (1RS)trans, $\alpha S$-cyano (1RS)-cis, $\alpha S$-cyano (1RS)-trans. This racemate is $\sim 3-4$-fold less lethal compared to $\alpha$-cypermethrin, a cypermethrin preparation with an isomer ratio of 1:1 $\alpha R$-cyano (1S)-cis, $\alpha$-cyano $(1 R)$-cis $[4,50-52,112,135,184]$.

In mammalian species (including humans), metabolism of pyrethrins and pyrethroids is rapid and occurs via ester hydrolysis or cytochrome P450-mediated oxidation [22,118, $70,100]$. In vivo toxicokinetic studies of orally administered pyrethroids in rats and mice have reported nearly complete elimination from the body in 2-8 days and half-lives in rat nervous system tissue in the range of $\sim 13-39$ h $[149,150$, $8,46,9]$. There is evidence that rates of detoxification are dependent upon the spatial conformation of pyrethroids $[167,22,75]$, which may contribute to the difference in potency observed between isomers. Thus, the isomeric composition of the pyrethroid preparation is a critical determinant of biological activity and must be considered when evaluating or comparing neurobehavioral studies.

\subsection{Structure-lethality relationships in rats}

Two major objectives have driven the development of pyrethroid chemical structures and selection of isomer compositions: namely, increases in both insecticidal potency and photostability $[48,91,125,126,156]$. Fig. 2 plots rat oral LD50s for pyrethroid compounds in chronological order by the approximate patent application date. Some of the milestones in the refinement of the pyrethroid structure are highlighted in the figure. Note that "modern" compounds (e.g., deltamethrin, esfenvalerate, $\alpha$-cypermethrin, $\lambda$-cyhalothrin, $\beta$-cyfluthrin, tefluthrin, bifenthrin, and imiprothrin), which are enriched in highly active stereoisomers, have far greater toxicity in rat than the "older" generations of pyrethroids. Even though the toxic potency of pyrethroids in rat has increased over time, the concurrent increase in insecticidal potency means that lower application rates may be sufficient for pest control, reducing the hazard posed by pyrethroids in non-target species such as mammals.

\section{Acute pyrethroid poisoning}

\subsection{Comparative toxicity between target and non-target species}

According to a general classification of behaviors elicited in insects by repellent and attractant chemicals, pyrethrum has been classically considered a locomotor stimulant, causing target individuals to disperse from a region faster than if the area did not contain the chemical $[42,57,15]$. More detailed studies demonstrate that in insects, and other invertebrates such as arachnids, pyrethroids produce reversible impairment of motor function and 'knockdown' in flying insect species that may be followed by death, depending upon the exposure level.

At low to intermediate levels of exposure, pyrethroids impair motor function in insects. This effect manifests as alterations in flying or walking patterns [6]. At intermediate dose levels, motor activity is disorganized, eventually forcing non-flying species to become immobilized and flying species to fall down $[152,115]$. Insect "knockdown" is comparable to the abrupt disruption of locomotor activity patterns seen in spiders after an acute sublethal exposure to cypermethrin [11]. Sublethal effects in insects, like incoordination and hyperactivity, may occur after acute exposure to about $1 / 10$ of an insect LD50 dose [15]. At higher exposure levels, the syndrome progresses from the initial period of hyperactivity and motor incoordination to tetanization, prostration, and convulsions followed by death [13].

Disruption of motor function is not the only effect of acute pyrethroid exposure in insects at sublethal doses [15]. For instance, rapid disorganization of calling behavior and chemical communication systems with consequent disruption of social/ sexual behaviors has been reported after sublethal exposure to deltamethrin in corn borers. In addition, calling behavior in deltamethrin-treated moths remained at $50 \%$ of control performance at 4 days post-dosing [192]. 


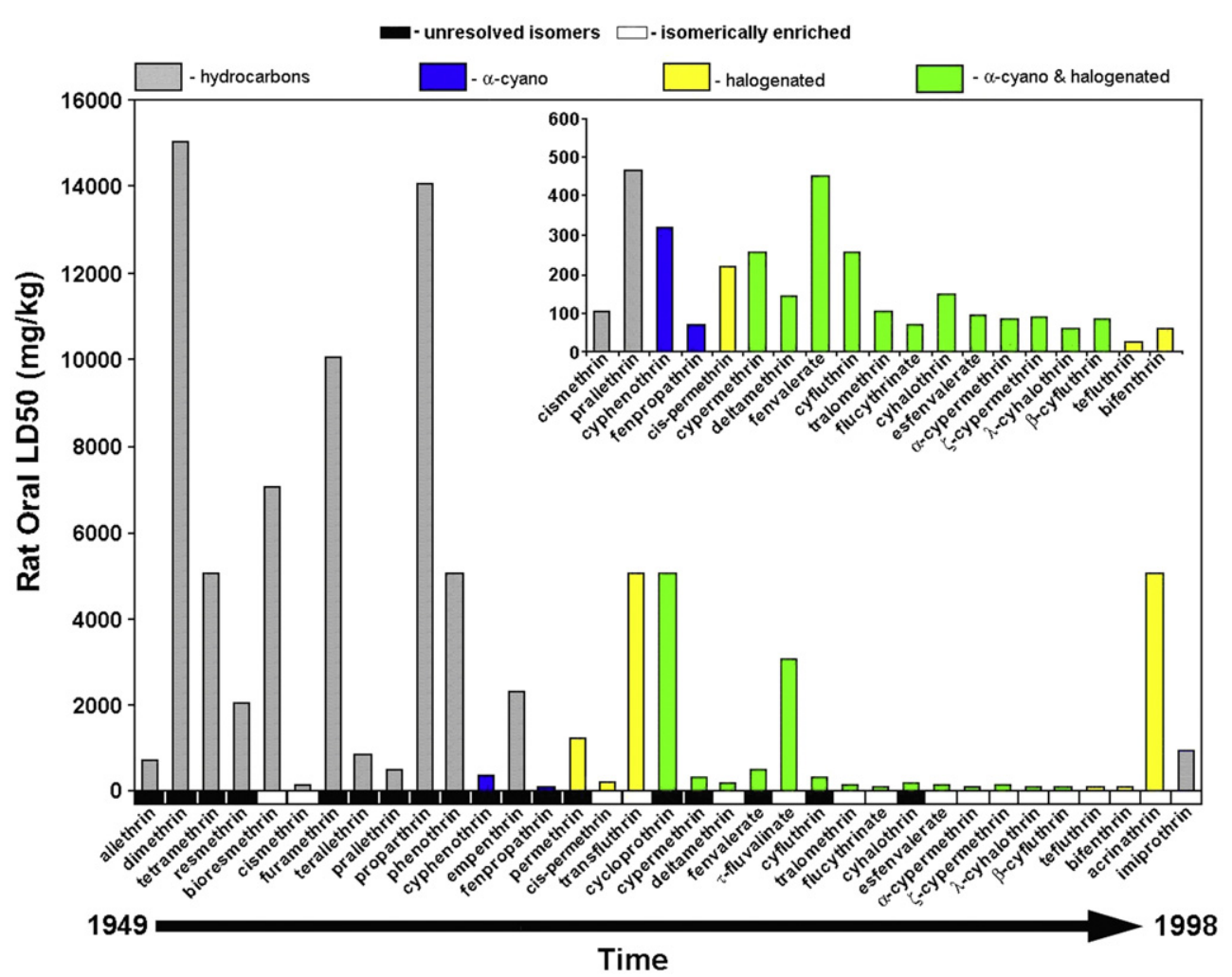

Fig. 2. Oral LD50s in rats following acute exposure to pyrethroids. Compounds are ordered chronologically according to the approximated date of the original patent application $[74,126,91,93]$. Only pyrethroids with central ester bonds are included. Milestones in the development of the structural properties of synthetic pyrethroids are denoted by the colored bars. Pyrethroid preparations that are isomerically enriched are denoted by white boxes lining the $x$-axis while formulations that are unresolved mixtures of many isomers are denoted by black boxes. All pyrethroids with LD50<500 mg/kg are pictured in the inset. Values for dimethrin, tetramethrin, bioresmethrin, phenothrin, empenthrin, and transfluthrin correspond to the maximal doses tested in literature studies, as true LD50s could not be determined. LD50s for a typical permethrin formulation (i.e., 40:60 cis:trans ratio) and a permethrin preparation enriched in cis-isomers (i.e., cis-permethrin) were taken from McGregor [112]. Taken from WHO [184]; PMRA [133]; WHO [181]; White et al. [180]; Miyamoto [118]; EC-HCPDG [46].

Pyrethroids are more selective for their target species as compared to other pesticide groups. Carbamates, organophosphates, and organochlorines have LD50 ratios (i.e., LD50 rat $_{\text {ins }} / \mathrm{LD} 50_{\text {insect }}$ ) below 100 while, in general, pyrethroids have LD50 ratios above $2000[48,172,91]$. However, when administered at a highly effective dose, pyrethroids produce evident neurotoxicity in nontarget species, including mammals. The signs of poisoning in nontarget species are similar in some respects to the severe signs of poisoning observed in insects $[68,47,15,172,45,84,76]$. These similarities may be attributed to similar molecular mechanisms-oftoxicity at target sites present across species, such as voltagesensitive $\mathrm{Na}^{+}$channels (VSSCs). In vitro studies have demonstrated that both insect VSSCs and a variety of mammalian VSSCs are sensitive to pyrethroids $[171,155,26]$. Differences in pyrethroid potency between species have been attributed to differences in 1) the intrinsic sensitivity of VSSCs to these compounds [171]; 2) body temperature $[180,60,137,65]$; and 3) metabolism $[118,67,148,70]$.

When first-pass metabolism in mammals is circumvented via intravenous (i.v.) or intracerebral (i.c.) administration of pyrethroids, the time course for the development of poisoning signs in insects and mammals is comparable. A latency of seconds to minutes from dosing to the onset of poisoning signs is seen in both cases $[98,62,127,13,139,39]$. In rodents, the onset of poisoning signs correlates with the accumulation of an internal dose in nervous system tissue [180,70,66,74,146]. Brain concentrations required to produce poisoning signs are comparable following administration by the oral or i.v. routes, even though the administered doses using the former route are much higher than those used in the latter $[92,180]$. This indicates that the detoxifying capacity for pyrethroids is dependent on route, and that metabolism and/or limited absorption play a significant protective role against pyrethroid neurotoxicity in mammals [156]. The signs and progression of acute pyrethroid poisoning observed in small rodents are further described in Sections 3.2 and 3.3, respectively.

Few data exist concerning pyrethroid-induced signs of poisoning in larger mammalian species. Some clinical veterinary reports describe the intoxication of dogs and cats by pyrethroids; however, these reports refer mostly to dermal exposures. The features of pyrethroid poisoning are similar across the mammalian species examined. Based on veterinary observations, acute, sublethal exposure to pyrethroids induces restlessness and hyper-excitability followed by "drunken movements" (i.e., locomotive ataxia), mydriasis, diarrhea, and general depression. In some cases motor incoordination, paresis, head bobbing, chewing, hyper-salivation, and/or whole-body tremors were reported [157,130,45,44,121,99]. 
Nevertheless, neurotoxicity observed in veterinary applications should not be assumed to have a direct dose-response relationship with the pyrethroid content of commercial formulations. While these data indicate that pyrethroid poisoning syndromes are comparable between rodent species and large mammals, no direct comparison of sensitivity across species can be determined from these reports due to the confounding influence of solvents, metabolic inhibitors, and other "inert" compounds in the commercial formulations used (see comparisons of toxicity in small rodents exposed to different formulations of deltamethrin, in Lepeshkin et al. [101], and of permethrin and fenvalerate in Williamson et al. [186]). Additional studies using technical grade pyrethroid formulations and comparable exposure routes are needed to establish accurate scaling factors across mammalian species. Lastly, there are very few data from quantitative evaluations of acute neurotoxicity in humans and primates following acute exposures to well-defined pyrethroid formulations. This precludes making direct comparisons between the extensive compendium of laboratory rodent studies available in the peer-reviewed literature and clinical reports in humans. Therefore, only data from pyrethroid studies using laboratory rodents are reviewed in the following sections.

In conclusion, even though mammalian species appear to be less susceptible to pyrethroid-induced neurotoxicity than insects, safety concerns will persist due to the similar neurotoxicity of these compounds across species after reaching an active target tissue dose.

\subsection{Signs of pyrethroid poisoning in rodents and classification schemes}

There has been a historical tendency to refer to individual pyrethroids as belonging to subcategories that were defined in early studies of the clinical signs of acute poisoning in rodents. A study by Verschoyle and Aldridge [175] describes signs of toxicity in rats for 36 pyrethroid preparations after i.v. administration of near-lethal to lethal doses. Their classification scheme separates 34 of the 36 tested pyrethroid preparations into two groups of compounds producing either whole-body tremors (T-syndrome) or choreoathetosis and salivation (CS-syndrome). The remaining two preparations produced mixed effects (i.e., tremor and salivation) and were termed TS-compounds. A later study by Lawrence and Casida [98] using i.c. injection of 29 pyrethroids outlined a toxicological classification scheme in mice that divided the tested compounds into two groups termed Type I and Type II, which correspond to the T- and CS-syndromes described in rat, respectively. A detailed comparison of the clinical signs observed in small rodents following exposure to both types of pyrethroids is shown in Table 1 . The key features of these syndromes are similar between rodent species regardless of the route of administration: i.c., i.v., i.p., or oral $[175,98,127,36]$. Generally, pyrethroids with a halogenated acid esterified to an $\alpha-$ cyano-3-phenoxybenzyl alcohol produce the CS/Type II syndrome. Pyrethroids lacking either or both of these moieties tend to produce the T/Type I syndrome. The historical classification of pyrethroids into T-, CS-, or TS-compounds $[175,98]$ does not

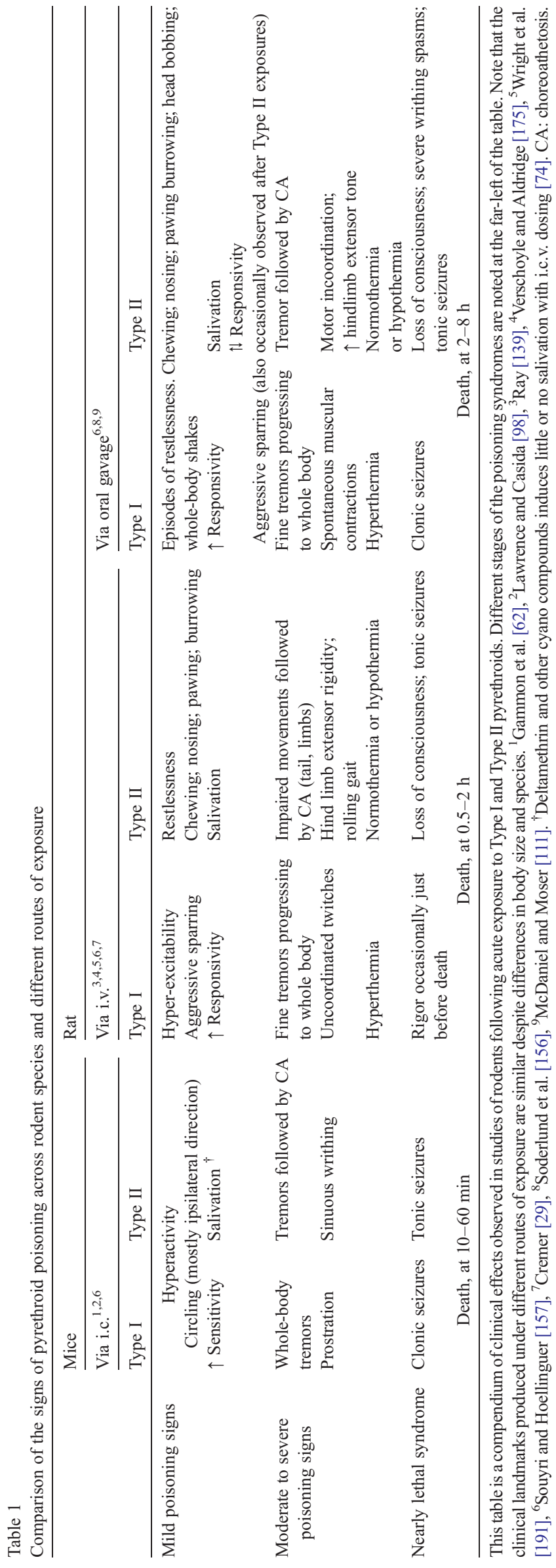


include a number of "modern" compounds that have been developed and registered since these experiments were conducted, i.e. bifenthrin, tefluthrin, fenfluthrin, acrinathrin, imiprothrin, and preparations enriched in highly active isomers such as $\beta$ cyfluthrin, $\lambda$ - and $\gamma$-cyhalothrin, and $\alpha$-cypermethrin. In the literature and the rest of this review, these unclassified pyrethroids are tentatively referred to as either CS- or T-syndrome compounds based on the muscular diskinesia described in more recent rodent studies (if available) or upon consideration of structural similarity to unequivocally classified compounds.

Exceptions to the aforementioned classification scheme have been noted. Natural pyrethrum extract and some non-cyano Type I pyrethroids (i.e., permethrin, transfluthrin, and tefluthrin) may produce salivation in rodents $[182,53,156]$. Fenpropathrin (an $\alpha$-cyano pyrethroid) produces muscular fasciculations more closely related to body tremors than the Type II landmark choreoathetosis $[175,5,98,23]$. Save for a few exceptions, the T(Type I)/CS(Type II)/TS(Type I/II) classification scheme holds true for poisoning syndromes induced by a majority of pyrethroids.

A study conducted by McDaniel and Moser [111] in LongEvans rats is the first to describe the distinctive (i.e. typespecific) neurobehavioral syndromes produced by permethrin and cypermethrin using a Functional Observational Battery (FOB). Multiple tests were performed to quantify changes over time in several endpoints 3-48 h following an acute, singledose, oral exposure to three doses of each pyrethroid (i.e. permethrin, 25-150 mg/kg, and cypermethrin, 20-120 mg/kg) in corn oil vehicle. For both compounds the highest dose tested was nearly lethal. Cypermethrin caused salivation and urination, splayed limbs, choreathetosis, increased foot splay, resistance to removal from home cage, decreased arousal, and decreased response to touch or tail pinch. Permethrin produced wholebody tremors, ataxia, greater resistance to removal from home cage, and increases in arousal, handling reactivity, and the response to touch or experimenter approach. Both compounds produced alterations in righting reflex, decreases in motor activity and grip strength, and increased click response. These observations were dose-related in most cases and, for most domains, consistent with the behavioral landmarks reported by other authors on exposure to non-cyano (Type I) and cyano (Type II) compounds in rat $[141,83,175,81,156]$.

Other researchers have explored the utility of alternative endpoints to classify pyrethroids. Wright et al. [191] used anesthetized rats to classify pyrethroids based on the number of abnormally evoked EMG spikes in the gastrocnemius muscle, and the presence or absence of abnormal trigeminal motor nucleus spikes. After-potential time constants were also measured in perfused isolated diaphragm preparations. Neurotoxic actions of compounds producing T- (i.e., fenfluthrin, cismethrin, and NRDC 108) and CS- (fluorocyphenothrin, cyhalothrin, cyfluthrin, fenvalerate, and deltamethrin) syndromes were clearly distinguished based on this characterization of neuromuscular function. Two compounds that evoke intermediate pyrethroid behavioral syndromes (i.e., cyphenothrin and fenpropathrin) also displayed intermediate electrophysiological profiles [191]. Grouping pyrethroids according to electrophysiological actions on skeletal muscle in rat is mostly consistent with previous pyrethroid classifications in rats (i.e., T-, CS- and intermediate TS-syndromes) and mice (i.e., Type I, Type II and intermediate Type I/II syndromes) [175,98,156].

Additional studies of effects of pyrethroids on phenobarbitalinduced sleep $[164,165]$ or sensorimotor function ([35,111] see Section 4.5) suggest that alternative criteria could be used for grouping of pyrethroid compounds. An alternative three-group classification system may also be derived using structure alone: in addition to the $\alpha$-cyano and non-cyano ester pyrethroids, a third subclass of compounds without an ester linkage can be established. The tremorgenic pyrethroid ethofenprox is an example of this third type [86,91]. There is currently scant knowledge concerning the toxicity of non-ester pyrethroids in mammals.

From a toxicological standpoint, the divergent neurobehavioral syndromes produced by different pyrethroids strongly suggest that different mechanisms of action are mediating the acute effects observed after "high-dose" exposures. The behavioral observations conducted 20-30 years ago (e.g. $[175,98,127])$ support this assumption. The T(Type I)/CS (Type II)/TS(Type I/II) classification scheme established in these studies is still useful for systematic comparison of acute poisoning syndromes between older and "modern" pyrethroids and across different insecticide classes. However, the practical application of this classification scheme for partitioning pyrethroids into different mechanistic categories for the purpose of human risk assessment is questionable. This is because the effective dose levels (i.e. nearly lethal) used to establish the $\mathrm{T}-\mathrm{CS}-\mathrm{TS}$ scheme are well above those that are relevant to human exposures. Neurobehavioral data from studies using doses that do not produce severe intoxication are more useful in the partitioning of pyrethroids into mechanistic subcategories. This "low-dose" range is much closer to environmentallyrelevant human exposures, allows for the establishment of no observable adverse effect levels (NOAELs), and allows generation of dose-effect data with greater pyrethroid-specificity while excluding functional responses that result from excessive physical stress (e.g. prolonged dehydration, energy exhaustion, seizures) that nearly lethal exposures may evoke. The studies presented in subsequent sections include data from "high-dose" exposures that supports the T/Type I-CS/Type IITS/Type I/II classification as well as dose-effect and timecourse data from studies using more environmentally-relevant exposures. Data from these latter studies either support or refute the partitioning of pyrethroids into different mechanistic categories depending on the neurobehavioral domain examined.

\subsection{Progression of acute poisoning syndromes}

The progression of pyrethroid-induced poisoning syndromes over time can be divided into five stages, with several distinct reversible alterations at sublethal doses, and an irreversible stage at very high doses. These stages were originally described for the progression of a CS-syndrome following i.p. injection of $40 \mathrm{mg} / \mathrm{kg}$ deltamethrin in LAC-Porton female rats. Behavioral signs appeared between 39 and 139 min after injection [141]. This 
time course typifies the progression of signs in mammalian species, which appear within $1 \mathrm{~h}$ of sublethal oral exposure, peak within $8 \mathrm{~h}$, and recover in 12-48 h [127,131,33,36,159,44,15, $121,99,111,156]$.

Table 2 summarizes the neurobehavioral findings for a typical Type I syndrome, focusing on the progression of whole-body tremors as observed by Nishimura et al. [127] after oral, i.v., or intracerebroventricular (i.c.v.) exposure of ddY male mice to allethrin. Both the route of exposure and the individual susceptibility of the test subject may influence the progression of poisoning signs [127,26,36,39]. During i.v. administration, fine tremors commence immediately after dosing and very little interindividual variability in the manifestation of signs is observed. Tremor intensity and duration increase in a dose-dependent manner at sublethal i.v. doses of allethrin. Tremors were rated mild, moderate, and severe in intensity at $5.56,6.67$, and $8 \mathrm{mg} / \mathrm{kg}$, respectively, indicating a steep dose-response. The tremorgenic action of allethrin was of longer duration at $8 \mathrm{mg} / \mathrm{kg}$ (i.e., $35-40 \mathrm{~min}$ ) as compared to $5.56 \mathrm{mg} / \mathrm{kg}$ (i.e., $15-20 \mathrm{~min}$ ). These results are consistent with the descriptions reported by Ray et al. [143] after i.v. administration of $S$-bioallethrin (i.e., an allethrin formulation enriched in trans-isomer) in rats. In contrast, the i.c.v. and oral routes required a longer time (1 to a few minutes) for clinical signs to manifest and the doseresponse relationships were more gradual than the i.v. route $[127,159]$. Other pyrethroids (i.e. cismethrin, cis-permethrin, and deltamethrin) have also been reported to produce milder and delayed poisoning syndromes when using i.c.v. administration as compared to the i.v. route [74]. It has not been determined yet whether this disparity is due to a difference in the time needed to reach threshold tissue levels in the brain, or the accumulation of an active dose in potential target tissues outside the brain (e.g. PNS; see [156]) from compound present in the circulation.

\section{Quantitative endpoints}

The following is a comprehensive compilation of reports on quantitative assays of pyrethroid neurobehavioral effects. The available peer-reviewed literature includes data from multiple endpoints that are presented in neurobehavioral domains as follows: motor activity, coordination, neuromuscular response, tremors, acoustic startle response, learning and memory, sensory response, and social interaction, reactivity to handling, anxiety, and sexual behavior. Information on the experimental designs and purity of test materials is included to allow for appropriate comparison of findings across studies. A clear disparity between the amounts of available evidence across neurobehavioral domains will be noticed.

\subsection{Motor activity}

Motor function is a behavioral domain impaired by all pyrethroid compounds regardless of species (see Tables 1-3). Before presenting any quantitative data on pyrethroid effects, some terminology will be defined as follows. Spontaneous motor activity refers to movements measured in the absence of any intervention (i.e., social, pharmacological, surgical, or environmental) influencing the willingness of the animal to move during the testing period and is usually applied to homecage observations of activity. Motor activity (or general motor activity) is the summation of movements in any part of the body regardless of the distance traveled in horizontal or vertical direction, in response to the testing conditions. Thus, motor activity includes locomotor and non-locomotor movements. Locomotor activity refers to ambulation, i.e. a change in the coordinate position of the subject within the testing device (open arena, maze, alley, etc.) by walking, circling, running, or rearing. Non-locomotor activity refers to behaviors such as scratching, pawing, grooming, burrowing, head or body shakes, and sniffing. Quantitative measurements of motor activity are the result of the interactions between the test subject and the testing apparatus. These interactions are recorded as patterns of motor-activity measurements that correspond to complex behaviors exhibited by the experimental animal throughout the testing trial in response to a novel environment. These include exploration, habituation, motivation, anxiety, and fear $[161,32,37]$. Administration of a test chemical may effectively modify these interactions, which would in turn result in

Table 2

Comparison of the time course of allethrin-induced neurotoxicity after exposure by different routes

\begin{tabular}{|c|c|c|c|c|c|}
\hline \multicolumn{2}{|l|}{ i.v. } & \multicolumn{2}{|l|}{ i.c.v. } & \multicolumn{2}{|l|}{ p.o. } \\
\hline $\operatorname{Time}^{\dagger}$ & Clinical sign & Time $^{\dagger}$ & Clinical sign & Time $^{\dagger}$ & Clinical sign \\
\hline Immediate & Fine tremor & $2-3$ & Fine tremor & 6 & Lacrimation \\
\hline $1-2$ & WBTs increase in severity & $3-7$ & WBTs increase in severity & $13-15$ & Fine tremor, retching and convulsive WBTs \\
\hline \multirow[t]{2}{*}{$2-3$} & \multirow[t]{2}{*}{ Tremors reaches peak intensity } & \multirow[t]{2}{*}{$7-9$} & \multirow[t]{2}{*}{ Tremor reaches peak of intensity } & $28-40$ & $\begin{array}{l}\text { Lying down ("reclining"), writhing w/severe tremors; } \\
\text { then hyper-sensitive to sound and touch }\end{array}$ \\
\hline & & & & 40 & Lying down ("being supine"), with severe WBTs for $\sim 2.5 \mathrm{~h}$ \\
\hline \multirow[t]{2}{*}{$4-16$} & \multirow[t]{2}{*}{ Decrease in tremor intensity } & \multirow[t]{2}{*}{$8-9$} & \multirow[t]{2}{*}{ Decrease in tremor intensity } & 190 & Trying to stand up, some tremor attenuation \\
\hline & & & & 225 & Motor incoordination and still some fine tremor \\
\hline \multirow[t]{2}{*}{$20-40$} & \multirow[t]{2}{*}{ Recovery of tremors } & \multirow[t]{2}{*}{$18-24$} & \multirow[t]{2}{*}{ Complete recovery } & 240 & Feeble tremors \\
\hline & & & & 360 & Partial recovery; prostration \\
\hline
\end{tabular}

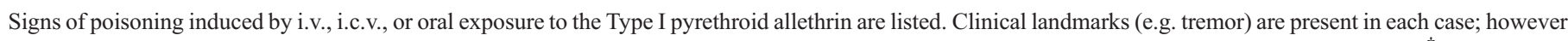

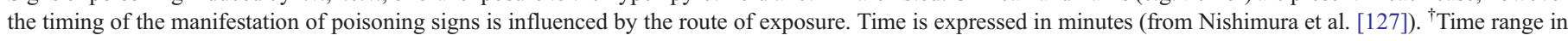

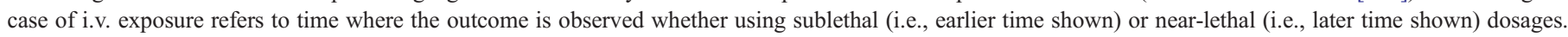
Abbreviatures: n.r. $=$ not reported; WBT $=$ whole-body tremor. 
Table 3

Effects of Type I and Type II pyrethroids on figure-eight maze activity

\begin{tabular}{|c|c|c|c|c|c|}
\hline Compound & Type & $\begin{array}{l}\text { Purity } \\
(\% \mathrm{w} / \mathrm{w})\end{array}$ & Time before testing (h) & $\begin{array}{l}\text { ED30 } \\
(\mathrm{mg} / \mathrm{kg})\end{array}$ & Ref. \# \\
\hline \multirow{5}{*}{ Deltamethrin } & \multirow[t]{16}{*}{ II } & $\mathrm{nr}$ & 2 & $\sim 3.7$ & 1 \\
\hline & & $\mathrm{nr}$ & 2 & $\sim 2.2$ & 2 \\
\hline & & $97+$ & 2 & $\sim 2.3$ & 3 \\
\hline & & $\mathrm{nr}$ & 2 & $\sim 1.8$ & 4 \\
\hline & & 98.9 & 2 & 2.51 & 7 \\
\hline \multirow[t]{3}{*}{ Cypermethrin } & & $\mathrm{nr}$ & 1.5 & $\sim 27$ & 5 \\
\hline & & 97 & 3 & $\sim 14$ & 6 \\
\hline & & 88 & 1.5 & 10.7 & 7 \\
\hline Fenvalerate & & $\mathrm{nr}$ & 1.5 & $\sim 15$ & 5 \\
\hline Esfenvalerate & & 98.6 & 2 & 1.20 & 7 \\
\hline Cyfluthrin & & $\mathrm{nr}$ & 2 & $\sim 8$ & 5 \\
\hline$\beta-C y f l u t h r i n$ & & 99.2 & 2 & 2.21 & 7 \\
\hline$\lambda$-Cyhalothrin & & 87.7 & 2.5 & 1.32 & 7 \\
\hline Fluvalinate & & $\mathrm{nr}$ & 2 & $\sim 9$ & 5 \\
\hline Flucythrinate & & $\mathrm{nr}$ & 2 & $\sim 2$ & 5 \\
\hline RU-26607 & & $\mathrm{nr}$ & 2 & $\sim 3$ & 5 \\
\hline Fenpropathrin & $\mathrm{I} / \mathrm{II}$ & 91.8 & 2 & 7.70 & 7 \\
\hline Cismethrin & I & $\mathrm{nr}$ & 1.5 & $\sim 10$ & 4 \\
\hline Resmethrin & & 92.3 & 4 & 292.8 & 7 \\
\hline$S$-bioallethrin & & 95.6 & 2 & 90.5 & 7 \\
\hline \multirow[t]{3}{*}{ Permethrin } & & 95.0 & 4 & $\sim 68$ & 6 \\
\hline & & $\mathrm{nr}$ & 1.5 & $\sim 90$ & 5 \\
\hline & & 92.0 & 1.5 & 42.7 & 7 \\
\hline Tefluthrin & & 92.6 & 2 & 2.26 & 7 \\
\hline \multirow[t]{3}{*}{ Bifenthrin } & & 89.0 & 4 & 3.21 & 7 \\
\hline & & & 4 & $5.19 *$ & 8 \\
\hline & & & 4 & $11.69 * *$ & 8 \\
\hline
\end{tabular}

Oral ED30s for pyrethroid compounds tested in different laboratories under similar dosing conditions using the figure-eight maze. Data from adult, male, Long-Evans rats is shown save study \#1 where CD 1 males were used. ED30s were obtained using mathematical models in studies \#7-8. For the other studies, ED30 values are visual estimations obtained from the corresponding reports. All studies used $1 \mathrm{~h}$ as the testing period except study \#1 (2 h). Corn oil was used as vehicle in all studies and dose volume was $1 \mathrm{ml} / \mathrm{kg}$ except study \#4 $(0.2 \mathrm{ml} / \mathrm{kg})$ and study \#8 ( 1 or $5 \mathrm{ml} / \mathrm{kg}$; marked by $*$ or $* *$, respectively). Technical grade compounds were examined in each study.

$\mathrm{nr}=$ not reported. ${ }^{1}$ Reiter et al. [145], ${ }^{2}$ Gilbert et al. [64], ${ }^{3}$ Crofton et al. [36], ${ }^{4}$ Crofton and Reiter [33], ${ }^{5}$ Crofton and Reiter [35], ${ }^{6} \mathrm{McD}$ aniel and Moser [111], ${ }^{7}$ Wolansky et al., [187], ${ }^{8}$ Wolansky et al., [189].

alterations in the pattern of motor activity observed during the testing phase. Thus, motor-activity measurements represent a "composite score" which may include changes in a number of unique behaviors rather than a change in a single, well-defined behavior.

We have recently determined the relative potencies of eleven pyrethroids in Long-Evans rats using motor activity in a figureeight maze [187]. Relative potency factors (i.e., for each compound $i$, ratio $\mathrm{ED} 30_{\mathrm{IC}}{ }^{1} / \mathrm{ED} 30_{i}$ where $\mathrm{IC}$ is the index compound), ranged from 0.0086 (resmethrin) to 2.09 (esfenvalerate), using deltamethrin as the index compound. ${ }^{1}$ Based on

\footnotetext{
${ }^{1}$ Deltamethrin has one of the most complete databases for neurobehavioral effects in rat [156], including extensive dose-response and time-course data using motor activity in a figure-eight maze or open field as an endpoint (see Appendix A). Motor activity measurements across a number of deltamethrin studies have demonstrated reproducible estimates of potency [145,33,34,64,36,187]. Moreover, its toxicokinetics in small rodents is one of the best studied among pyrethroids $[8,156,116,70]$.
}

this work and other quantitative motor-activity assessments conducted following acute, oral exposure to pyrethroids using corn oil as vehicle and $1 \mathrm{ml} / \mathrm{kg}$ as dose volume [145,33,35,111, $36,187,189$ ] several generalizations can be made (see Table 3):

i) All pyrethroids tested (seventeen pyrethroid preparations) produce dose-dependent decreases in locomotor activity.

ii) Compounds enriched in highly active isomers have greater potency than unresolved preparations, though the overall effect (i.e., decrease in motor activity) is the same regardless of isomer composition.

iii) On average, $\alpha$-cyano compounds are about 10 -fold more potent than non-cyano compounds in decreasing motor activity.

iv) Pyrethroids display up to a $\sim 240$-fold difference in relative potencies for motor activity. Variability in potency among non-cyano compounds was much greater than variability among cyano compounds (i.e., 127-fold and 21-fold, respectively).

v) Generally, pyrethroids produce a rapid progression of neurotoxicity. Within $24 \mathrm{~h}$ after dosing, onset and complete recovery of control-like performance is observed.

vi) Decreases in activity occur at dosages producing typical pyrethroid signs (e.g., salivation, tremors, and choreathetosis) and below. ED30 doses for motor activity were found at levels well below the corresponding LD50s; e.g., for deltamethrin, $\lambda$-cyhalothrin and esfenvalerate, ED30s were 27-, 42-, and 73-fold lower, respectively [187].

At sublethal doses, complete recovery of control-like motor activity occurs within $8 \mathrm{~h}$ of dosing in most cases. $S$-bioallethrin has a rapid progression in a motor-activity assay (peak at $0.5-$ $1 \mathrm{~h}$; full recovery within $3 \mathrm{~h}$ ) while bifenthrin has a slower progression with a persistent decrease in motor activity and tremors lasting 8-24 h, depending upon dosing conditions [80,156,187,189].

Other laboratories, using a variety of testing devices, have reported decreases in motor activity following acute oral exposure to permethrin [82], cyhalothrin [2], $\lambda$-cyhalothrin, $[81,138]$, fenvalerate $[41,105]$, cypermethrin [81], and deltamethrin [25] that are consistent with the effects listed above. No cumulative effect of pyrethroids on locomotor activity in rats was observed following repeated (once daily) oral exposure for 30 days to deltamethrin $(2 \mathrm{mg} / \mathrm{kg})$ and cismethrin $(6 \mathrm{mg} / \mathrm{kg})$ in corn oil [33].

Appendix A (see supplementary data in URL link) summarizes eighteen studies of pyrethroid-induced effects on motor activity including assays that used commercial formulations as test materials. While most laboratories have observed decreases in activity, a limited number of studies have demonstrated slight increases or no effects on locomotor activity following pyrethroid exposure. However, these studies used commercial products with a high mass-percentage of solvents or "inerts" [38], low-absorption routes of exposure (i.e. dermal; Mitchell et al. [117]) or long post-dose intervals prior to motor-activity measurement [90]. These factors and the rapid 
toxicokinetics of the compounds used $[63,112,116]$ may have contributed to the anomalous results in these studies.

A degree of caution must be exercised in interpreting doseresponse data for pyrethroids in studies using automated photobeam-based testing devices. Photoelectric devices (i.e. open arenas or mazes) are designed to measure photobeam interruptions in individually tracked photocells. Counts can be registered by both locomotor and non-locomotor behaviors. In our experience testing a wide variety of pyrethroids with an extensive range of sublethal doses [33,35,36,187-189], the proportion of photobeam interruptions collected at any given photocell is consistent across cells at doses that decrease total motor activity but do not produce overt poisoning signs. In contrast, a marked alteration of the distribution of photobeam interruptions across photocells may be expected in a test subject undergoing severe signs of pyrethroid intoxication due to repetitive movements in front of a single photocell (i.e. prolonged tremor or choreoathetosis, seizures) and the inability to ambulate across adjacent photocells. In this situation, interpretation of the collected data may be misleading. The difficulties in extrapolating from a nearly lethal dose producing virtual absence of locomotion due to exhaustion and prostration to a "low-dose" producing a decrease in ambulation due to mild gait impairment can exemplify this problem. Thus, pyrethroid doses that produce excessive toxicity in the test subject should be avoided when generating dose-response data using an automated photobeam-based test to insure increased accuracy and precision of potency estimates for the test compound.

In summary, a number of pyrethroids from each clinical type (i.e., T-, CS- and TS-syndrome-causing compounds) have been evaluated in the open field or in maze-like devices and, in general, a dose-related decrease of general motor activity was found. Examination of a variety of pyrethroid structures supports motor activity as a simple and sensitive measure of toxicity for the entire pyrethroid class. This endpoint has been used to: 1) determine the relative potencies of numerous, structurally diverse pyrethroid preparations, 2) define threshold doses for pyrethroid toxicity [187], 3) successfully predict the effect of exposure to pyrethroid mixtures [188], and 4) explore the influence of oral dosing volume on pyrethroid potency [189] in rodent models.

At this time, motor activity is the most extensively characterized neurobehavioral endpoint for pyrethroid effects. Nevertheless, motor activity is an apical measure of functional impairment that may be affected by an array of diverse chemicals through a variety of mechanisms $[110,144,95]$. Other endpoints that may provide specific information on the mechanism(s) of pyrethroid toxicity should be used in conjunction with motor activity when determining the relationship between internal dose, target tissue level, and adverse neurobehavioral effects in the context of generating relevant information for estimating hazard [169,128]. Even though motor activity lacks specificity, this endpoint may provide complementary information to neurobehavioral assays that have been proposed to have specificity for different pyrethroid types such as startle response and tremorgenic action $[33,35,111,156$, 189]. Thus, data from dose-response assays for motor activity will aid in the interpretation of findings for mechanism-specific endpoints.

\subsection{Coordination}

In addition to effects on motor activity, pyrethroids also affect other behaviors that are dependent upon the coordinated movement of the animal. Decreases in motor coordination in the rotarod test were observed following $0.5-1.5 \mathrm{~g} / \mathrm{kg}$ deltamethrin in mice [25], $14.5-145 \mathrm{mg} / \mathrm{kg}$ deltamethrin in rats [108], and $15-150 \mathrm{mg} / \mathrm{kg} \alpha$-cypermethrin in rats [107]. Chanh and coworkers used latency to fall from the rod as measure of effect and gum-arabic solution as dosing vehicle, while the later two studies used the number of falls from the rotating rod during the full test period $(1 \mathrm{~min})$ as the measure of effect and DMSO as dosing vehicle. Cypermethrin and deltamethrin have similar structures, produce CS-syndromes, and evoke similar alterations on motor and sensorimotor endpoints $[33,35,111$, 187,156]. The rotarod studies further support the similarity between the neurotoxicological profiles of cypermethrin and deltamethrin.

Quantitative effects on righting reflex (i.e., using a FOB severity scoring method) have been reported for permethrin and cypermethrin in male and female Long-Evans rats. These data indicate that coordination is markedly impaired after oral exposure to $150 \mathrm{mg} / \mathrm{kg}$ permethrin (though only males showed statistically significant effects) or $60-120 \mathrm{mg} / \mathrm{kg}$ cypermethrin (for both males and females) in corn oil [111]. Qualitative or semi-quantitative descriptions of pyrethroid-induced deficits in balance and gait are also present in the literature for a number of T- and CS-syndrome compounds, e.g., bifenthrin, $S$-bioallethrin, permethrin, $\beta$-cyfluthrin, $\lambda$-cyhalothrin, cypermethrin, $\zeta$-cypermethrin, $\alpha$-cypermethrin, and deltamethrin $[156,106,108]$.

In summary, even though limited comparison across studies could be conducted due to differences in dosing and testing conditions, the accumulated evidence strongly suggests that pyrethroids, regardless of type, affect motor coordination. Since the above motor coordination studies do not include sufficient dose-response data, estimation of threshold doses for this endpoint is not possible. Dose-response data using one of the alternative tests proposed to quantify motor coordination deficits $[169,183,128,158]$ and a few compounds of each type would help in determining if this endpoint is more sensitive than others in detecting pyrethroid-induced neurotoxicity. Lastly, like motor activity, coordination in the rotarod- and rightingreflex assays may be affected by a variety of compounds and similar outcomes may result from a variety of physiological or toxicological conditions $[140,169,183,128]$, precluding a direct association between effect measurements and pyrethroidspecific mechanism(s) of toxicity.

\subsection{Neuromuscular response}

A number of pyrethroid compounds have been evaluated using grip strength as a behavioral endpoint. A grip strength test utilizing a strain gauge designed to measure neuromuscular response was included in the FOB study conducted by 
McDaniel and Moser using Long-Evans rats (see introduction of this study in Section 3.2). The authors observed a dosedependent decrease in both forelimb and hindlimb grip strength of adult rats 3-4 h after an acute oral dose of 25$150 \mathrm{mg} / \mathrm{kg}$ permethrin or $20-120 \mathrm{mg} / \mathrm{kg}$ cypermethrin in corn oil (see Table 4). Structurally, these chemicals are identical save for the addition of an $\alpha$-cyano group to the alcohol moiety of cypermethrin (Fig. 1). Moreover, a similar FOB protocol was recently applied to characterize the neurotoxicity of bifenthrin (i.e., a potent T-syndrome pyrethroid) in corn oil in adult rats. A dose-related weakening of forelimb grip strength was observed after oral administration of $1-20 \mathrm{mg} / \mathrm{kg}$ bifenthrin [187], which is consistent with the effects of permethrin [111], another T-syndrome pyrethroid.

In another study, supermethrin (i.e., a commercial formulation of cypermethrin) was examined using a grip strength assay $4 \mathrm{~h}$ after acute exposure of male rats [81]. Four doses of supermethrin (i.e., 4.5, 9, 18, and $27 \mathrm{mg} / \mathrm{kg}$ ) were tested using oral gavage route and corn oil as vehicle. Consistent with the results of McDaniel and Moser [111], supermethrin weakened the response in this assay. Interestingly, the lowest dose producing significant effects compared to control was different for hind- and forelimb grip tests (i.e., 9 and $27 \mathrm{mg} / \mathrm{kg}$, respectively). Differential sensitivity of hind- and forelimb grip responses (i.e., hindlimb grip more sensitive than forelimb grip for cypermethrin neurotoxicity) was also observed by McDaniel and Moser [111] in the case of female rats but not in males. It is unknown why differential sensitivity of hind- and forelimb measurements of grip strength was observed in male rats in one study and females in the other. Other studies have demonstrated a decline in grip strength in rats following oral exposure to natural pyrethrins $[50,51]$ and synthetic pyrethroid preparations including $\tau$-fluvalinate [49], bifenthrin, $S$-bioallethrin, $\beta$-cyfluthrin, deltamethrin [156], and fenvalerate [113].

A study by Abou-Donia et al. [3] also noted a reduction of grip strength in rats on days 30 and 45 after dermal administration of technical grade permethrin in $70 \%$ ethanol $(0.13 \mathrm{mg} / \mathrm{kg} / \mathrm{day})$. This study used a route of exposure that results in lower blood concentrations of pyrethroids than the oral route $[124,163]$. Also, in contrast to the study of McDaniel and Moser [111], latency (i.e., time to release a wood dowel gripped with both forepaws) was the endpoint. This is an indirect measurement of grip strength. Differences in these experimental conditions prevent direct comparison of the two studies.

One comprehensive toxicological evaluation of $\alpha$-cypermethrin did not show any significant effects on grip response after acute oral (gavage) exposure or repeated oral (dietary) exposure of CRL:CD rats to doses up to $40 \mathrm{mg} / \mathrm{kg}$ and $27 \mathrm{mg} / \mathrm{kg} / \mathrm{day}$, respectively [52]. In the acute exposure assays, rats were tested $5 \mathrm{~h}$ after administration of a single oral dose. In the repeated dose neurotoxicity assessments, rats were tested during weeks 2 and 6 of a 6 -week study. Isomerically enriched $\alpha$-cypermethrin is approximately three-fold more toxic than cypermethrin $(\mathrm{CYP})$ by the oral route (i.e., $\mathrm{LD} 50_{\mathrm{CYP}} / \mathrm{LD} 50_{\alpha-\mathrm{CYP}} \approx 3$ ) in rats [184]. The effective acute oral doses of cypermethrin cited by McDaniel and Moser [111] for inducing decreases in grip strength were $60-120 \mathrm{mg} / \mathrm{kg}$. Thus, multiple experimental and biological factors may have contributed to disparate results observed between these two cypermethrin studies.

In conclusion, the available evidence strongly suggests that pyrethroids, regardless of clinical profile, weaken the neuromuscular response as observed in grip strength assays conducted using rats. Like the previously described endpoints (i.e. motor activity and coordination), there is a consistent decrease in response for all the pyrethroid compounds examined. However, the database is insufficient to determine the relative sensitivity of this endpoint for pyrethroid neurotoxicity. Finally, decreases in grip strength observed following acute pyrethroid exposure do not seem to provide a means to distinguish compounds that produce T, CS, or TS poisoning syndromes. In summary, this endpoint would not improve the precision, simplicity, or sensitivity observed in studies using motor activity (see Table 3 ) as an outcome [136,111,35,187].

\subsection{Tremors}

Tremors are one of the most consistent neurobehavioral signs following exposure to overtly toxic doses of pyrethroids. Although several studies have qualitatively described pyrethroid-induced tremors in animals, only a handful have quantitatively examined this effect. Two methods have been employed to quantify pyrethroid-induced tremors: 1) rankordered scales of severity and 2) spectral analysis.

Table 4

Effects of Type I and Type II pyrethroids on neuromuscular function

\begin{tabular}{|c|c|c|c|c|c|c|c|c|}
\hline \multirow[t]{4}{*}{ Functional measure } & \multicolumn{4}{|c|}{ Permethrin } & \multicolumn{4}{|c|}{ Cypermethrin } \\
\hline & \multicolumn{2}{|l|}{ Males } & \multicolumn{2}{|c|}{ Females } & \multicolumn{2}{|l|}{ Males } & \multicolumn{2}{|c|}{ Females } \\
\hline & \multicolumn{2}{|c|}{ Dose (mg/kg) } & \multicolumn{2}{|c|}{ Dose (mg/kg) } & \multicolumn{2}{|c|}{ Dose (mg/kg) } & \multicolumn{2}{|c|}{ Dose $(\mathrm{mg} / \mathrm{kg})$} \\
\hline & 75 & 150 & $75^{\dagger}$ & 150 & 60 & 120 & 60 & 120 \\
\hline Forelimb grip & $71 \%$ & $75 \%$ & $101 \%$ & $77 \%$ & $65 \%$ & $53 \%$ & $84 \%$ & $28 \%$ \\
\hline Hindlimb grip & $70 \%$ & $64 \%$ & $85 \%$ & $79 \%$ & $54 \%$ & $53 \%$ & $67 \%$ & $44 \%$ \\
\hline Mean limb grip effect & $70.5 \%$ & $69.5 \%$ & $93 \%$ & $78 \%$ & $59.5 \%$ & $53 \%$ & $75.5 \%$ & $36 \%$ \\
\hline
\end{tabular}

Dose-response relationships on grip strength for one Type I (i.e., permethrin) and one Type II compound (i.e., cypermethrin). Both pyrethroids were administered in corn oil by the oral route $(1 \mathrm{ml} / \mathrm{kg}$ ). Testing was conducted at 3 (cypermethrin) or 4 (permethrin) h post-dosing. Grip strength data is expressed as percentage of vehiclecontrol. For both compounds, cis- and trans-isomers were present in similar quantities. For permethrin ( $95 \%$ pure), a total recovery of control-like performance was observed at $24 \mathrm{~h}$ post-dosing. For cypermethrin (97\% pure), grip strength depression was still evident $24-48 \mathrm{~h}$ after exposure (taken from McDaniel and Moser [111]). ${ }^{\dagger}$ All measurements of hindlimb and forelimb grip strength were significantly different from control except for females at $75 \mathrm{mg} / \mathrm{kg}$ permethrin. 
Nishimura et al. [127] scored the progression of tremors in mice following exposure to the T-syndrome pyrethroid allethrin by three separate routes: i.c.v. $(0.34-0.91 \mathrm{mg} / \mathrm{kg})$, i.v. (5.56$8 \mathrm{mg} / \mathrm{kg}$ ) and p.o. (480 mg/kg). The actions of allethrin were not scored in the same fashion across the different routes of exposure examined. In the i.v. study, the authors developed a four-point scale for tremor severity, spanning "light tremors of the body but not limbs" (score of 0.5 ) to "lying down and writhing with severe tremors" (score of 2). For the i.v. exposure, dose-dependent increases in tremor severity or incidence were observed [127]. For i.c.v. administration, the variation in tremor magnitude across doses was minor, so a simple designation of presence or absence of tremor throughout the body was used. Tremors observed following oral administration were not scored.

The FOB study by McDaniel and Moser [111] also noted that the incidence and intensity of permethrin- and cypermethrininduced tremors increased in a dose-dependent manner following an oral exposure. The characteristics of the tremors differed between the two types (strictly clonic movements with permethrin, clonic and tonic movements with cypermethrin). In this work, tremor was noted as 'absent', 'mild', 'moderate', or 'severe'. The occurrence of moderate to severe whole-body tremor was observed at 2-4 h after exposure to permethrin (i.e. $75-150 \mathrm{mg} / \mathrm{kg}$ ), and increased in intensity in a dose-related fashion. Cypermethrin (i.e. $60-120 \mathrm{mg} / \mathrm{kg}$ ) greatly affected equilibrium and muscle tone, and produced spasmodic (i.e. choreathetotic) flexions and extensions of the body musculature that were noticeable at $1.5,3$ and $24 \mathrm{~h}$ post-dosing.

Another study using scores for tremor severity was recently conducted by Anand et al. [10]. This study evaluated adolescent (40 days old) and adult (90 days old) male SD rats $(\mathrm{N}=4$ per group) that were orally administered $10 \mathrm{mg} / \mathrm{kg}$ deltamethrin in glycerol formal. Treated rats were observed at $1 \mathrm{~h}$ intervals for up to $6 \mathrm{~h}$ after dosing. Tremors were ranked using a $0-1-2-3$ scale, spanning from no tremor (score of 0 ) to violent tremor and no voluntary control of movements (score of 3 ). An equivalent scale was used for ranking the intensity of salivation in the same animals. No evident tremor was observed. However, both age groups had a rapid onset of mild-to-moderate salivation that peaked at $2 \mathrm{~h}$ and recovered by 3-4 $\mathrm{h}$ after exposure. Transient tremors rapidly progressing to choreoathetosis after acute exposure to deltamethrin have been previously described in the literature; this is a neurobehavioral sign that occurs after the onset of salivation and only appears at doses higher than those that produce mild-to-moderate salivation [141,146,16,191]. Since severe salivation was not observed in this study, the absence of tremors was likely due to a relatively low dose of deltamethrin under the dosing conditions used.

In the second type of study, Herr et al. [77] conducted a spectral analysis of permethrin-induced tremors $5 \mathrm{~h}$ after an oral dose of $120 \mathrm{mg} / \mathrm{kg}$. Tremor intensity was quantified using a load cell transducer attached to a platform where the rat was placed, and was expressed as a power curve along a range of frequencies (i.e., 2.5-22.5 Hz). Permethrin-treated animals showed higher peak amplitudes across frequencies than vehiclecontrols. The magnitude of this difference was particularly large in the $12.5-22.5 \mathrm{~Hz}$ range. Permethrin was also examined by Hudson et al. [83] using a similar method. In this study, the time course of permethrin-induced tremor was characterized for doses of 120 and $240 \mathrm{mg} / \mathrm{kg}$. For both doses, tremor was undetectable at $1 \mathrm{~h}$ and reached a peak at $5 \mathrm{~h}$. Total recovery was observed at $12 \mathrm{~h}$ and $24 \mathrm{~h}$ after 120 and $240 \mathrm{mg} / \mathrm{kg}$, respectively. In addition, tremors were further quantified at $5 \mathrm{~h}$ after exposure to 45,90 , and $180 \mathrm{mg} / \mathrm{kg}$ permethrin. Tremor intensity increased in a dose-dependent manner at 90 and $180 \mathrm{mg} / \mathrm{kg}$. Spectral analysis of tremor frequency has not been used to examine other pyrethroid compounds. Therefore, amplitude changes induced by permethrin within a particular frequency range should not be considered a specific result that is appropriate for measuring the tremorgenic action of all T-syndrome pyrethroids, as other compounds may induce peak effects in different frequency ranges. Moreover, the frequencies at which peak amplitude (i.e., peak intensity) of tremor is observed cannot be assumed a priori to be the same between doses. This complicates the selection of the appropriate frequency range for measuring tremor within and across compounds. Notably, in the same work, measurements of startle response amplitude and rectal temperature were more sensitive than tremor intensity for detecting permethrin-induced effects [83], which provides evidence that spectral analysis of tremors could be less sensitive than other methods for quantifying pyrethroid neurotoxicity.

Future studies of tremor using spectral analysis should include dose-response relationships for a number of pyrethroid compounds. Additional tremor assays would help determine 1) whether peak effects are consistently observed in the same frequency range for multiple compounds; 2) whether this endpoint has the sensitivity required to construct relative potency relationships; and 3) whether peak response amplitudes would remain in the same frequency range across doses. If tremor patterns were different between compounds, then the capability of this measure to distinguish between different pyrethroid subclasses $[175,98,111,156]$ makes tremor pattern a useful (i.e., specific) endpoint for creating a partitioning criteria for these insecticides.

\subsection{Acoustic startle response}

The acoustic-evoked startle response (ASR) is a behavioral endpoint that has been used to study pyrethroid effects on sensorimotor function in rats $[33,35,79,78]$. Contrary to the consistent pattern of motor-activity effects (i.e., decrease in activity), divergent effects on ASR parameters (i.e., latency and amplitude) have been observed dependent upon the structure of the pyrethroid. Table 5 shows the results obtained with several Type I and II compounds evaluated under similar dosing and testing conditions (taken from Crofton and Reiter, [33,35]). Type II compounds with phenoxybenzyl alcohols linked to a halogenated acid moiety (i.e., cypermethrin, deltamethrin and cyfluthrin) produce a decrease in ASR amplitude and an increase in latency. However, Table 5 also shows that Type II compounds with acid moieties that contain aromatic rings (i.e. fenvalerate, fluvalinate, and flucythrinate) do not produce the 
Table 5

Effect of Type I and Type II pyrethroids on acoustic-evoked startle response (ASR)

\begin{tabular}{|c|c|c|c|c|c|}
\hline \multirow{2}{*}{$\begin{array}{l}\text { Pyrethroid } \\
\text { name }\end{array}$} & \multirow[t]{2}{*}{ Type } & \multirow{2}{*}{$\begin{array}{l}\text { Dose range } \\
(\mathrm{mg} / \mathrm{kg})\end{array}$} & \multicolumn{3}{|c|}{ Effect on startle response parameters } \\
\hline & & & Latency & Sensitization & Amplitude \\
\hline$p, p$-DDT & N/A & $50-75$ & No & $\downarrow$ & $\uparrow$ \\
\hline Cismethrin & I & $6-18$ & No & $\downarrow$ & $\uparrow$ \\
\hline Permethrin & I & $60-120$ & No & $\downarrow$ & $\uparrow$ \\
\hline RU11679 & I & $15-30$ & No & $\downarrow$ & $\uparrow$ \\
\hline Cypermethrin & II & $37-150$ & $\uparrow$ & $\downarrow$ & $\downarrow$ \\
\hline Deltamethrin & II & $2-6$ & $\uparrow$ & $\downarrow$ & $\downarrow$ \\
\hline Cyfluthrin & II & $25-75$ & $\uparrow$ & $\downarrow$ & $\downarrow$ \\
\hline Flucythrinate & II & $3-15$ & $\uparrow$ & No & No \\
\hline Fluvalinate $^{\dagger}$ & II & $25-150$ & $\uparrow$ & No & No \\
\hline Fenvalerate & II & $10-40$ & No & $\uparrow$ & $\uparrow$ \\
\hline
\end{tabular}

Single doses of nine pyrethroids were administered in corn oil by the oral route $(1 \mathrm{ml} / \mathrm{kg})$. All compounds except fluvalinate modified the startle response amplitude. The organochlorine pesticide $p, p$-DDT was also included because of its similar action at voltage-sensitive channels as compared to Type I pyrethroids [122]. Contrary to the common actions of all pyrethroids on motor function (i.e. activity decrease), qualitative differences were observed among different pyrethroid compounds using ASR as the endpoint. The doses examined in these ASR studies are in the same range that produces evident decreases in motor activity in the figure-eight maze $[33,35,111,187]$. Taken from Crofton and Reiter, [33,35]. 'The main effect of treatment was significant (ANOVA, $p<0.05$ ), but post hoc pairwise comparisons did not reach statistical significance between control- and fluvalinate-treated groups. ${ }^{\star}$ There was a trend for an increase in startle response latency that did not reach statistical significance.

same ASR alterations, indicating only a partial commonality in ASR effects across cyano compounds. Type I compounds (i.e. permethrin, cismethrin, RU11679) increase ASR amplitude and have no effect on latency $[33,35]$. These results are mostly consistent with those reported by Hijzen and co-workers for permethrin and deltamethrin $[78,79]$.

These results, obtained using quantitative ASR testing systems, are only partially consistent with those found in semi-quantitative or qualitative assessments of sensorimotor response following acute exposure to pyrethroids. Part of the FOB implemented by McDaniel and Moser [111] generated severity scores for click-, touch-, and approach-responses at 3 or $\sim 5 \mathrm{~h}$ after oral dosing with cypermethrin and permethrin, respectively. Touch- and approach-response scores were decreased with cypermethrin or increased with permethrin; however, the click-evoked response was increased for both compounds [111]. In another study, rats were assessed for click response using FOB procedures after oral exposure to $1-20 \mathrm{mg} /$ $\mathrm{kg}$ of the T-syndrome pyrethroid bifenthrin in corn oil [189]. A dose-related increase in click response scores was observed from $6-20 \mathrm{mg} / \mathrm{kg}$. This finding is consistent with abovementioned ASR [35] and FOB [111] results for permethrin, a prototypic " $T$ " compound. Nevertheless, equivalent cypermethrin preparations and animal models produced opposite effects on two closely related endpoints across studies, i.e., the ASR [35] and the click response [111]. Moreover, Hijzen and Slangen [78] report a dose-dependent increase in startle amplitude in rats following oral exposure to cypermethrin $(60-120 \mathrm{mg} / \mathrm{kg})$ in corn oil. In this study, oral exposure to deltamethrin $(6 \mathrm{mg} / \mathrm{kg})$ in corn oil attenuated ASR amplitude, a finding consistent with the result of Crofton and Reiter [33] using the same vehicle. The reasons for the inconsistency between laboratories and across compounds (i.e., equivalent findings for permethrin and deltamethrin, opposite changes found for cypermethrin) are unclear (see Table 5).

Sensorimotor observations were also included in the extensive study of 29 compounds carried out by Lawrence and Casida [98] in mice (see details in first paragraph, Section 3.2). Increases in startle response are mentioned as a general finding but no compound-specific information for this aspect of pyrethroid neurotoxicity was provided in this report. There are other descriptions of unpredictable patterns of sensitivity to external stimuli, with no clear distinction between cyano and non-cyano compounds [156]. Unfortunately, the diversity of species, routes, and dosing/testing procedures used to assess sensorimotor output complicates a direct comparison across above-mentioned pyrethroid studies.

A synthesis of ASR and click-response findings for pyrethroids shows that there is an evident alteration of sensorimotor function after pyrethroid exposure, although a few exceptions to this rule may exist (i.e., see fluvalinate in Crofton and Reiter [35]). Some contradictory findings highlight the difficulties in interpreting the structure-specific actions of pyrethroids in this behavioral domain. Examination of a greater number of T-, CS- and TS-syndrome compounds in standardized time-course and dose-response assays would help determine if the acoustic startle response upholds the T-CSTS (or Type I-II-I/II) classification scheme for partitioning pyrethroid compounds into groups with similar adverse effects in mammals.

A tentative grouping criterion for ASR patterns could be proposed based on the available data. Pyrethroids could be divided as follows: T-syndrome compounds producing an increase in startle amplitude (i.e., permethrin, bifenthrin, cismethrin, and RU11679), CS-syndrome compounds producing a decrease in startle amplitude (i.e., deltamethrin, cypermethrin, cyfluthrin, and flucythrinate), and a third (minor?) group of pyrethroids (i.e., fenvalerate) that produces alterations in ASR parameters that are not predicted by the presence or absence of a cyano moiety and are not dependent on the type of clinical landmarks observed. The utility of divergent acoustic startle response profiles in partitioning pyrethroids into clinical categories has not yet been examined beyond the semiquantitative FOB assays conducted by McDaniel and Moser [111]. Examining neuronal responses along the acoustically evoked startle reflex axis using electrophysiological recordings and/or pharmacological challenges, coupled with additional neurobehavioral characterization of this functional domain (see $[151,31,160,134])$, could be useful in determining the utility of this domain for elucidating the neurological basis of divergent pyrethroid effects.

\subsection{Learning and memory}

The acute effects of pyrethroids on learning and memory have been examined in a number of studies. In previous sections, data were presented that motor and sensorimotor behaviors are altered by pyrethroid doses well below those 
producing evident whole-body tremor, choreoathetosis, or convulsions. The accumulated evidence indicates that learning, and memory tasks that are dependent upon motor or sensorimotor function may also be disrupted by pyrethroids at levels well below those that induce severe neurotoxicity. The available reports focused on two different assays to assess pyrethroid effects on learning: schedule-controlled operant behavior (using food as a reward) and aversively motivated learning.

Pyrethroid-induced neurobehavioral effects were characterized using schedule-controlled behavior as an endpoint in rats and mice. Peele and Crofton [132] utilized this approach with adult rats exposed orally to permethrin $(100-400 \mathrm{mg} / \mathrm{kg}$ ) or cypermethrin $(7.5-60 \mathrm{mg} / \mathrm{kg})$ in corn oil. The response rate (lever press) following a visual stimulus was measured in operant conditioning chambers under a variety of variable interval reinforcement schedules. Both pyrethroids impaired performance, with some compound-specific differences. Cypermethrin (at doses up to $30 \mathrm{mg} / \mathrm{kg}$ ) did not modify the theoretical maximal response rate (MR), although a dose-dependent decrease in overall response rate was observed. Administration of $7.5,15$, and $30 \mathrm{mg} / \mathrm{kg}$ cypermethrin increased the reinforcement rate required to maintain half-maximal responses in a dose-dependent manner with no effect on MR, suggesting decreased motivation. Moreover, cypermethrin at $60 \mathrm{mg} / \mathrm{kg}$ affected both $\mathrm{MR}$ and the relationship between reinforcement rate and performance. It was concluded that cypermethrin affects motivational mechanisms that influence the association between reinforcement rates and motor response [132]. In addition, a clear dose-dependent decrease in overall response rates was observed with permethrin at dosages of $200-400 \mathrm{mg} / \mathrm{kg}$. However, in the case of permethrin at $300-400 \mathrm{mg} / \mathrm{kg}$, an equivalent decrease in response rates (i.e., $37-61 \%$ drop) was associated with only a $15-36 \%$ decrease in reinforcement rates, indicating no major role of motivation on permethrin-evoked deficit in performance. These findings suggest differences in the mechanisms of toxicity responsible for the decrease in operant performance occurring after exposure to permethrin and cypermethrin for which the only structural difference between them (i.e., presence of an $\alpha$-cyano group in cypermethrin) would play a determinant role.

Table 6 summarizes literature reports that use operant behavior as an endpoint. Data from these studies indicate that a variety of pyrethroids decrease operant response rates, regardless of reinforcement schedule in rats $[103,14,132,159]$ and in mice [69]. Noteworthy, in the study of Glowa [69], no evident change in the deltamethrin $(3 \mathrm{mg} / \mathrm{kg})$-induced decrease in responding rates was observed after repeated (once daily) administration for 10 days compared to the decrease observed $30 \mathrm{~min}$ after the first single dose. This is consistent with the absence of cumulative effects after repeated exposure to deltamethrin in rat using motor activity as an endpoint ([33]; see p.21). In addition, permethrin modifies schedule-controlled behavior at lower doses $(30-60 \mathrm{mg} / \mathrm{kg})$ using i.p. exposure [14,159] as compared to $200-400 \mathrm{mg} / \mathrm{kg}$ using the oral route [132]; however, differences in experimental conditions other than route of exposure could have produced this disparity of effective dose ranges. This table also includes a report in which permethrin (15-60 mg/kg i.p.) failed to modify acquisition of a

Table 6

Acute effects of Type I and Type II pyrethroids on schedule-controlled operant response

\begin{tabular}{|c|c|c|c|c|c|c|c|c|}
\hline & Species & $\begin{array}{l}\text { Route/ } \\
\text { Vehicle }\end{array}$ & $\begin{array}{l}\text { Operant response } \\
\text { schedule }\end{array}$ & $\begin{array}{l}\text { Dose-test } \\
\text { interval }\end{array}$ & $\begin{array}{l}\text { Pyrethroid } \\
\text { used }\end{array}$ & $\mathrm{I} / \mathrm{II}$ & $\begin{array}{l}\text { Dose range } \\
(\mathrm{mg} / \mathrm{kg})\end{array}$ & Outcome \\
\hline \multirow[t]{4}{*}{$\begin{array}{c}\text { Bloom et al. } \\
{[14]}\end{array}$} & \multirow[t]{4}{*}{ SD rat } & \multirow[t]{4}{*}{$\begin{array}{l}\text { i.p./EM }{ }^{\mathrm{a}} \\
(2 \mathrm{ml} / \mathrm{kg})\end{array}$} & \multirow[t]{4}{*}{$\mathrm{VI}, 20 \mathrm{~s}, \mathrm{R}=$ food pellet } & \multirow[t]{4}{*}{$20 \mathrm{~min}$} & Permethrin $^{c}$ & I & $15-60$ & $\begin{array}{l}\text { Dose-related } \downarrow \\
\text { in response }\end{array}$ \\
\hline & & & & & cis-Permethrin & I & 30 & $\downarrow$ Operant response \\
\hline & & & & & trans-Permethrin & I & 30 & No effect \\
\hline & & & & & Deltamethrin & II & 2 & $\downarrow$ Operant response \\
\hline Glowa [69] & $\begin{array}{l}\text { Crl:CD1 } \\
\text { mice }\end{array}$ & $\begin{array}{l}\text { i.p./EM }{ }^{\mathrm{b}} \\
(5 \mathrm{ml} / \mathrm{kg})\end{array}$ & FI, $60 \mathrm{~s}, \mathrm{R}=25 \mu \mathrm{lmilk}$ & $\begin{array}{l}\mathrm{CD} \text {, sessions begin } \\
\text { after each dose }\end{array}$ & Deltamethrin & II & $0.02-3.0$ & $\downarrow$ Operant response \\
\hline $\begin{array}{l}\text { MacPhail et al. } \\
\text { [103] }\end{array}$ & LE rat & $\begin{array}{l}\text { p.o. } / \mathrm{CO} \\
(1 \mathrm{ml} / \mathrm{kg})\end{array}$ & $\begin{array}{l}\mathrm{FR}, 40 \mathrm{R}=50 \mu \mathrm{l} \text { milk offered } \\
\text { after } 14 \text { th lever press }\end{array}$ & $120 \mathrm{~min}$ & Deltamethrin & II & $1-8$ & $\begin{array}{l}\text { Dose-related } \downarrow \\
\text { in operant response } \\
\uparrow \text { Latency before } \\
\text { responding }\end{array}$ \\
\hline \multirow{2}{*}{$\begin{array}{l}\text { Peele and Crofton } \\
\text { [132] }\end{array}$} & \multirow[t]{2}{*}{ LE rat } & p.o./CO & $\mathrm{VI}, 10 / 30 / 90 / 270 \mathrm{~s}$ & \multirow[t]{2}{*}{$90 \mathrm{~min}$} & Permethrin & I & $100-400$ & $\downarrow$ Operant response \\
\hline & & $(1 \mathrm{ml} / \mathrm{kg})$ & $\mathrm{R}=$ food pellet & & Cypermethrin & II & $7.5-60$ & $\begin{array}{l}\text { Dose-related } \downarrow \\
\text { in operant response }\end{array}$ \\
\hline \multirow[t]{4}{*}{ Stein et al. [159] } & \multirow[t]{4}{*}{ SD rat } & \multirow[t]{4}{*}{$\begin{array}{l}\text { i.p./EM }{ }^{\mathrm{a}} \\
(2 \mathrm{ml} / \mathrm{kg})\end{array}$} & \multirow[t]{4}{*}{$\mathrm{VR}, 25 \mathrm{R}=$ food pellet } & Immediate & $(R)$-trans-allethrin & I & $8-32$ & $\begin{array}{l}\text { Dose-related } \downarrow \\
\text { in operant response }\end{array}$ \\
\hline & & & & $20 \mathrm{~min}$ & Permethrin $^{\mathrm{c}}$ & I & $15-60$ & $\downarrow$ Operant response \\
\hline & & & & $20 \mathrm{~min}$ & Deltamethrin & II & $1-3$ & $\downarrow$ Operant response \\
\hline & & & & $20 \mathrm{~min}$ & Fenvalerate & II & $5-7$ & $\downarrow$ Operant response \\
\hline $\begin{array}{l}\text { Van Haaren et al. } \\
\text { [174] }\end{array}$ & SD rat & $\begin{array}{l}\text { i.p./EM }{ }^{\mathrm{a}} \\
(2 \mathrm{ml} / \mathrm{kg})\end{array}$ & LEOR R = food pellet & $\begin{array}{l}15 \text { min; cumulative } \\
\text { response over } 8 \mathrm{~h}\end{array}$ & permethrin & I & $15-60$ & No effect \\
\hline
\end{tabular}

The effects of pyrethroids on operant responding using a variety of experimental designs. Details on animal models, test materials, dose ranges, and testing conditions are included in the table. Three to five dose levels were tested in most cases. The absence of effects using trans-permethrin [14] is consistent with the generally poor toxicity observed in pyrethroid preparations enriched in trans-isomers in small rodents [156]. ${ }^{a}$ Emulphor diluted with ETOH and $9 \%$ saline, ${ }^{b}$ emulphor in sterile water. Permethrin isomer ratio was 40:60, cis:trans $^{\mathrm{c}}$, or minimum 35\% cis, maximum 65\% trans ${ }^{\mathrm{d}}$. Abbreviations: Species: Long-Evans (LE), Sprague-Dawley (SD). Route/Vehicle: intraperitoneal (i.p.), oral (p.o.), emulphor (EM), corn oil (CO). Operant Response Schedule: reward (R), variable interval (VI), fixed interval (FI), variable ratio (VR), fixed ratio (FR), light-evoked operant response (LEOR), reinforcer (R), cumulative dose (CD). 
light-evoked response (lever press) in male and female rats [174]. It remains uncertain how the dosing and testing conditions of this study may have resulted in this finding.

Pyrethroids have been shown to decrease the rate of food intake $[92,182,81]$. This is a potential confounder in evaluating pyrethroid-induced decreases in operant response rates in rats where food is the reinforcer (see e.g., [159]). However, the anorexic effects of pyrethroids occur at or above the highest doses used in these operant evaluations. Accordingly, it is unlikely that changes in food intake confounded operant response rates following the acute, low-dose exposures to pyrethroids used in the studies summarized in Table 6.

Little evidence is available for aversively motivated learning. Husain et al. [85] reported that repeated oral exposure to a commercial formulation of deltamethrin ( $7 \mathrm{mg} / \mathrm{kg} /$ day, 15 days) in rats caused a 30\% decrease in a re-learning index for an avoidance response based on visual discrimination in a Y-maze $24 \mathrm{~h}$ after the last deltamethrin dose. In addition, a deficit in an avoidance behavior task was reported in adult rats treated for 20 days with $18 \mathrm{mg} / \mathrm{kg} /$ day of a commercial formulation of $\lambda$-cyhalothrin using oral gavage administration [81]. In the same study, $4.5-27 \mathrm{mg} / \mathrm{kg}$ of a commercial cypermethrin formulation (i.e., "supermethrin") had no effect in the same test, in either the training or the retention phases [81]. However, these assays are not treated in detail here because the effects were observed days to weeks after repeated doses of commercial pyrethroid formulations, thus making comparisons with the acute effects of technical grade pyrethroids difficult.

Assessments of schedule-controlled behavior and aversive learning may provide a very sensitive method for detecting pyrethroid-induced neurotoxicity in rat. Permethrin given at an oral dose of $7.5 \mathrm{mg} / \mathrm{kg}$ (i.e. 1/6 of the oral ED30 for motor activity; Wolansky et al. [187], effectively modifies several parameters of schedule-controlled responding [132]). Reiter et al. [145] describe oral ED50s for deltamethrin using aversive learning (i.e., $1.6 \mathrm{mg} / \mathrm{kg}$ ) or schedule-controlled conditioning (i.e., $3.5 \mathrm{mg} / \mathrm{kg}$ ) that are lower than that described in a motoractivity test (i.e., $7.6 \mathrm{mg} / \mathrm{kg}$ ).

In summary, a few T- and CS-syndrome pyrethroids have been evaluated using learning-related endpoints after acute exposures in small rodents. Most of the available evidence on these acute effects consistently shows that pyrethroids, regardless of type, produce dose-related decreases of operant response rates in tests involving food as a reward. As mentioned in previous sections, pyrethroids produce acute decreases in motor activity, weakening of neuromuscular strength, and impaired motor coordination. The relative role of these three effects in contributing to the deficits observed in tests of learning and memory using operant responding or aversive learning is uncertain. In addition, schedule-controlled behavior presents an apparent increase in sensitivity for detecting pyrethroid effects when compared to other neurobehavioral endpoints such as motor activity. However, these data were obtained using a variety of experimental conditions (i.e., different animal models, routes of exposure, dosing conditions, and testing procedures), restricting the generalizations that can be made when comparing studies.
As a tentative conclusion, scheduled controlled operant response and aversive learning tests may provide models to examine pyrethroid-induced deficits in motoric performance as well as learning and memory with acceptable sensitivity and specificity and allow the exploration of mechanistic differences between pyrethroids producing different syndromes. Interpretation of the available data (i.e., operant responding rates in particular) may be difficult due to its dependence upon the integrated performance of several neurobiological systems (e.g., motor coordination, sensory responses, and crossmodal association). In addition, the execution of schedule-controlled operant conditioning assays are generally more complex and time-consuming than most of the other neurobehavioral endpoints discussed in this review. Ultimately, the information on the pyrethroid-type specificity of these endpoints is limited and their apparently superior sensitivity (a clear advantage when examining the effects of environmentally-relevant lowlevel exposures) cannot be used yet as an argument to support learning and schedule-controlled operant responding as effective endpoints for classification of pyrethroid syndromes.

\subsection{Somatosensory response}

The FOB implemented by McDaniel and Moser [111] included semi-quantitative assays of touch-, click- and tailpinch responses at different times after oral exposure of rats to permethrin and cypermethrin. Both pyrethroids evoked changes in all of these endpoints (see Sections 3.2 and 4.5). The progression of these changes showed apparent similarity to those observed for other neurobehavioral endpoints (e.g., motor activity, gait, neuromuscular response, body temperature), with maximal effects occurring within $4 \mathrm{~h}$, and recovery by $24 \mathrm{~h}$ after exposure. Compared to other endpoints used in the same study, the reactivity to different sensory stimuli did not show any advantage in sensitivity for detection of pyrethroid neurotoxicity. However, opposite effects were found after permethrin and cypermethrin exposure (i.e., decrease or increase, respectively) in both touch and tail-pinch responses, suggesting some pyrethroid-type specificity of these sensory endpoints.

In addition, two studies used a guinea-pig flank model to characterize the effects of commercial insecticide formulations containing cyano and non-cyano pyrethroids on the skin sensory response following dermal exposure $[18,104]$. The authors counted the number of times the treated animals showed behavioral activity (i.e., episodes of licking, rubbing, scratching, and biting) directed to the side of the shaved back where the test pyrethroid had been administered compared to the activity directed to the vehicle-treated contralateral side of the back. An increase in activity on the pyrethroid-treated side was evident, and this behavioral response had a rapid progression (i.e., onset, peak, and recovery within the first $4 \mathrm{~h}$ after exposure). However, the observed behavioral signs re-appeared when a pharmacological challenge (i.e., oil of mustard) was administered to the pyrethroid-treated site $24 \mathrm{~h}$ later. Formulations containing cyano compounds (fenvalerate, cypermethrin, deltamethrin, and flucythrinate) produced a greater sensory stimulation than that including permethrin as active ingredient. Another laboratory 
[88,89] examined male mice after intrathecal (i.t.) administration of fenvalerate $(0.01-3 \mu \mathrm{g})$ using two measures of sensory response. First, the cumulative amount of time spent on nociceptive responses (i.e., biting, paw licking, and scratching episodes) was counted during a 30 -min session starting immediately after fenvalerate injection using acrylic observation chambers. Second, latency to withdrawal of the tail was measured after noxious heating of the skin using a light bulb under different voltage intensities. This tail-flick latency was determined before and $60 \mathrm{~min}$ after exposure to fenvalerate. A dose-related increase in the cumulative time of nociceptive episodes was observed along the entire dose range examined. In addition, tail-flick latency was decreased. Using a series of pharmacological assays (e.g., morphine), the occurrence of fenvalerate-induced allodynia/hyperalgesia was proposed by the authors $[88,89]$. No studies were found examining the acute effects of pyrethroid insecticides on vision, smell, hearing or other sensory modalities in laboratory rodents besides the studies mentioned above.

Few conclusions can be made for pyrethroid-induced neurobehavioral effects in the sensory domain. The limited data available are of little relevance for risk assessment purposes (i.e., effects assessed after i.t. injection) or confounded by the presence of highly concentrated non-pyrethroid ingredients in the test material (i.e., commercial formulations). In addition, only one pyrethroid of each type was examined using the FOB assays. Thus, the few datasets obtained under very different experimental conditions preclude a comprehensive analysis of the evidence across laboratories. Finally, it remains to be determined if the specificity of the touch and tail-pinch responses described after exposure to permethrin and cypermethrin [111] is consistent across a wider array of T- and CSsyndrome pyrethroids.

\subsection{Social interactions, reactivity to handling, anxiety, and sexual behavior}

A few studies are available on the acute effects of pyrethroids on social interaction between cagemates, reactivity to handling or response to removal from cage, and sexual behavior. Most of these studies did not conduct quantitative analyses of the reported observations. A distinction must be made between effects that are based on the primary actions of pyrethroids and those stemming from modified behaviors required for social and sexual interactions. For example, prominent motor dysfunction (e.g., decrease of motor activity and deficit of motor coordination) is expected to reduce the opportunities for expressing these interactions. The multiplicity of factors that influence social and sexual behaviors (i.e., motor performance, sensory thresholds, emotional reactivity, fear, and housing conditions) poses additional difficulties in the standardization of testing conditions for these endpoints within and across laboratories. Data interpretability is also an issue. Since there are common influences that affect these different behaviors and there is little quantitative information available on them for pyrethroids, the reports using these endpoints have been grouped in this section.
Verschoyle and Aldridge [175] observed that, after i.v. exposure, a number of pyrethroids evoke bizarre and aggressive behaviors in LAC-Porton female rats. These pyrethroids included cismethrin, bioresmethrin, phenothrin, permethrin, and fenpropathrin (i.e., T-syndrome compounds in most cases). Moreover, McDaniel and Moser [111] quantified the ease of removal from the home cage as part of their comprehensive neurobehavioral evaluation of permethrin $(25-150 \mathrm{mg} / \mathrm{kg})$ and cypermethrin $(20-120 \mathrm{mg} / \mathrm{kg})$ in Long-Evans rats of both sexes. Dose-dependent increases in scores for this endpoint were found in both cases. At highly effective doses, permethrintreated rats were very difficult to handle (a few showing aggressive behavior), whereas cypermethrin-treated rats did not show aggressive reactions in any case.

There is evidence from two studies that demonstrates that commercial formulations of CS-syndrome pyrethroids reduce the time spent in social interaction in male, adult Wistar rats $[41,2]$. The former study assessed acute effects in an open field arena $1.5 \mathrm{~h}$ after a single, oral exposure to fenvalerate [41]. The latter study used a similar observation protocol but the session was started $24 \mathrm{~h}$ after the last oral exposure to cyhalothrin under a 7-day repeated (once a day) dosing scheme [2]. The decrease in time spent in social interactions was shown to be dosagerelated for fenvalerate $(1-30 \mathrm{mg} / \mathrm{kg})$ but no dose-dependence was found for the effect of cyhalothrin $(1-7 \mathrm{mg} / \mathrm{kg})$. In addition, an increase in fighting episodes after oral exposure to $7 \mathrm{mg} / \mathrm{kg}$ deltamethrin (i.e., Decis ${ }^{\circledR}$, a commercial formulation containing $2.8 \%$ deltamethrin) under a 15 -day repeated (once a day) dosing scheme has been reported [85].

There is little and inconsistent evidence for the anxiogenic effect of CS-syndrome compounds in the rat. The two studies mentioned above for fenvalerate and cyhalothrin included elevated plus-maze assays [41,2]. Fenvalerate did not produce any acute effect on any parameter of anxiety using this test $1.5 \mathrm{~h}$ after oral exposure [41]. In contrast, cyhalothrin (at $3 \mathrm{mg} / \mathrm{kg}$, once daily during 7 days) evoked a $30 \%$ decrease in time spent in open arms, and a mild increase in time spent in closed arms [2], suggesting an anxiogenic-like response.

Last, only one study could be found quantifying the effects of a commercial pyrethroid formulation on various aspects of sexual behavior. Ratnasooriya et al. [138] administered a insecticide product (i.e., ICON ${ }^{\circledR}$ ) containing $10 \% \lambda$-cyhalothrin as the active ingredient to young adult albino rats of both sexes and carried out cage-side observations $0-5 \mathrm{~h}$ after dosing. Two doses of $\lambda$-cyhalothrin were prepared in distilled water (63 and $100 \mathrm{mg} / \mathrm{kg}$ ) and were administered daily, by gavage, for 1 week. Clinical signs appeared 2-3 h after dosing, and persisted for 6$10 \mathrm{~h}$. Treated males displayed an inhibition of sexual behaviors at the two doses examined. This effect included inhibition of a libido index, and increase in the latency for mounting, intromission, and ejaculation, suggesting an overall impact on sexual motivation. It is uncertain if these effects on sexual behavior were secondary to ICON ${ }^{\circledR}$ induced alterations on other functional domains.

In summary, the limited evidence available indicates that pyrethroid exposure may disrupt some aspects of social and sexual behaviors and may produce alterations in reactivity to 
physical contact with the experimenter or between cagemates in rodent species. However, the data are insufficient to determine whether these effects are observed for the entire pyrethroid class or if they are specific to compounds of the T, CS, or TS subclasses. It should be noted that a number of the studies included in this section used commercial formulations as test materials that include inert ingredients, precluding any definitive conclusions about the action of the tested pyrethroids on social and sexual behaviors and anxiety. For instance, the ICON ${ }^{\circledR}$ formulation used in the sexual behavior assay contains $90 \%$ "inactive ingredients" that have unknown effects on the examined endpoint. The conclusion that the active ingredient of $\mathrm{ICON}^{\circledR}$ (i.e., $\lambda$-cyhalothrin) is responsible for the alteration of sexual behaviors can only be made if a technical formulation containing $\lambda$-cyhalothrin is examined under the same testing conditions. The sparse quantitative data regarding aggression, handling, anxiety, social interactions and sexual behavior make the utility of these endpoints for risk assessment purposes uncertain.

\subsection{Other descriptions of pyrethroid-induced neurobehavioral toxicity}

The comprehensive FOB study that has already been mentioned in previous sections [111] shows acute, dose-related increases in the incidence of abnormal movements and gait impairment in male rats after a single oral exposure to permethrin or cypermethrin. The lowest dose that produced alterations in these endpoints was $75 \mathrm{mg} / \mathrm{kg}$ and $20 \mathrm{mg} / \mathrm{kg}$ for permethrin and cypermethrin, respectively. At the highest doses tested, cypermethrin $(120 \mathrm{mg} / \mathrm{kg})$ induced much greater gait abnormality scores than permethrin $(150 \mathrm{mg} / \mathrm{kg})$, even though the highest dose of permethrin produced moderate to severe whole-body tremors. This suggests that motor-related endpoints are more susceptible to cypermethrin-induced dyskinesia than permethrin-induced tremors. In addition, Hornychová et al. [81] carried out a comprehensive FOB-like battery of tests to examine supermethrin, a pyrethroid preparation derived from cypermethrin. This study used similar animal model (young adult rats) and dosing conditions (corn oil as vehicle and $1 \mathrm{ml} / \mathrm{kg}$ as dose volume) but different administration schedules (i.e., repeated daily dosing) and testing times (i.e., $4 \mathrm{~h}$ to 28 days after first dose). In general, for all functional domains evaluated during the acute phase of this complex evaluation the findings were consistent with the cypermethrin assays conducted by McDaniel and Moser [111]. Thus, only three pyrethroid preparations (i.e., permethrin, cypermethrin, and supermethrin) have been examined using FOB-like protocols $[111,81]$, so the limited available evidence precludes generalizations.

FOB assays are valuable tools for the screening of potential neurotoxicants; however, measurements are subjective [162]. Therefore, while studies using FOB-like protocols can provide comprehensive descriptions of chemical-induced neurotoxicity, the execution of the FOB needs to be conducted by well-trained experimenters to assure reproducibility within and between studies. In the case of pyrethroids, FOB could be applied for exploratory analysis and clinical classification of compounds under the proposed T-, CS- and TS-syndrome scheme. However, multiple functional observations using only a few dose levels may not be as advantageous as extensive doseresponse assays using a few specialized tests for defining sensitivity thresholds or estimating relative potencies for numerous pyrethroids $[162,169,183,128]$. A tentative analysis of the available FOB data shows that while the full FOB protocol includes numerous functional evaluations (mostly qualitative descriptions and ranked observations), in the cypermethrin study using male rats, motor activity (figureeight maze) and (rectal) body temperature were the most sensitive endpoints [111]. Therefore, FOB assays show a greater potential as a method to refine the toxicological classification of pyrethroids than as a functional evaluation to obtain potency estimates with superior sensitivity.

\section{Variables that influence pyrethroid potency in neurobehavioral studies}

In most cases, analysis of potencies for pyrethroids is complicated due to inconsistent results observed under different experimental conditions across studies. In many reports, similar animal models, test compounds, and dosing vehicles were used to examine comparable neurobehavioral domains. However, comparisons cannot be made because of inconsistent testing conditions or an incomplete description of experimental methods. Pyrethroid-induced neurotoxicity may be influenced by a variety of biological factors and/or experimental conditions $[68,74,156,23]$. Vehicle and route of exposure [127,26,36], dosing volume [189], isomer composition [156], formulation [186], age [154], and body size [122], are recognized determinants of pyrethroid toxicity. There is also emerging evidence suggesting that different test apparatus [37,32] and food deprivation [114] may also determine differences in the outcomes after acute exposure to pyrethroids. Last, circadian rhythms [196,140,179], ambient temperature [122] and housing conditions $[71,72]$, are additional conditions with potential to influence estimates of pyrethroid potency using neurobehavioral endpoints. Identification of the biological and experimental determinants of pyrethroid neurotoxicity and ascertainment of their actual impact on pyrethroid potency estimates would provide valuable guidance for the interpretation of neurobehavioral data collected in a variety of laboratory settings. The multiplicity of factors affecting endpoints for pyrethroid neurotoxicity advocates careful consideration of experimental conditions before elaboration of conclusions within or across studies.

\section{Significance of neurobehavioral findings and research needs}

This exhaustive and comprehensive compilation of data on the neurobehavioral effects of acute exposure to pyrethroid insecticides in adult mammals is intended to help plan the allocation of efforts and resources for expansion of the database for pyrethroid neurotoxicity, particularly in the context of identifying relevant information that can be used in risk 
assessment processes. Pyrethroid usage has increased throughout the last 20 years and will likely continue to increase in the future $[19,7,119,40,58]$. Notably, low-level exposure to multiple pyrethroids has been recently reported in human populations $[185,54,24,166,87]$. Given that simultaneous exposure to multiple pyrethroids occurs, a critical issue driving the current risk assessment efforts is whether or not to regulate pyrethroid compounds through a cumulative risk assessment framework, as individual compounds, or as two or more subclasses with divergent mechanisms $[94,156,153,142]$. One of the principal criteria for determining if a group of compounds should be examined in a cumulative risk format is that they act through the same mode-of-action [170]. Data on the neurobehavioral effects of pyrethroids can help guide policy decisions on which strategy is most appropriate for estimating human risk.

Based on in vitro data of nerve membrane and ion channel function, it has been proposed that all pyrethroid compounds act through the same primary mechanism of action $[123,122,156]$. In vitro studies such as these are of great value in identifying and characterizing the molecular sites of action for pyrethroids. However, data from neurobehavioral assays provide critical pieces of information not available from in vitro studies and yet essential to the primary goal of risk assessment, protecting human health, namely: 1) do the pharmacological actions of environmentally-relevant levels of different pyrethroids result in similar or dissimilar adverse outcomes, 2) which adverse effects are the most sensitive to pyrethroid exposure, 3 ) what are the exposure threshold levels for adverse effects produced by individual compounds, and 4) is the toxicity of environmentally-relevant pyrethroid mixtures equal to that produced by the mathematical additivity of the individual-compound effects? The current database on the neurobehavioral toxicology of pyrethroids can be used to begin to address these questions.

At this time, the accumulated neurobehavioral evidence summarized in this review both contradict and support mechanistic commonality for in vivo toxicity. If only functional observations of rats exposed to "high" sublethal levels of different pyrethroids were taken into account, the experimenter would conclude that the test compounds belong to at least two toxicological classes (see Table 1) due to the divergence of the acute poisoning signs that are observed (see also Crofton and Reiter [35]; McDaniel and Moser [111]; Peele and Crofton [132]). On the other hand, data obtained using a variety of neurobehavioral endpoints at "lower" doses indicate that there are similarities (motor activity, grip strength, motor coordination, and operant responding rates) and differences (startle and thermoregulatory responses, and some FOB-related measures) in the adverse effects observed across members of this pesticide class. Currently there is an effort to determine if all pyrethroid compounds act through the same toxicological mechanisms in intact mammals or if the action of different pyrethroids at primary (or secondary) molecular target sites results in activation of divergent toxicological pathways $[74,120,156$, 17,109]. A specific biochemical assay that can be used to predict pyrethroid-mediated neurotoxicity across mammal species (such as blood acetylcholinesterase inhibition in the case of organophosphate poisoning; B. Ryan et al. [12], and
USEPA [170]) is not available. In addition, the paucity of data concerning exposure of humans to minimally effective pyrethroid doses prevents determination of a critical effect (the adverse outcome observed at the lowest administered dose; USEPA [168]) or even whether or not the same neurobehavioral domains are sensitive to pyrethroid effects across species. In the absence of any other pyrethroid-specific assay, neurobehavioral effects observed at the "lower" end of experimentally effective dose ranges seem appropriate for use as partitioning criteria for different risk categories given that the administered doses are closer to the exposure levels observed in human populations. This strategy would be expected to increase the accuracy of NOAEL and threshold doses, and reduce the uncertainty related to the extrapolation from high-dose to low-level exposures in animals and the estimation of risk in humans using animal data.

\section{Summary and perspective}

Table 7 is a summary of the functional endpoints that have been used to explore pyrethroid-mediated effects on behavior and some physiological domains in the rat. Only reports including well-defined pyrethroid exposures (using test materials of technical purity) are considered. The number of compounds for which data have been reported in peer-reviewed sources is listed for each endpoint. In addition, this table includes a comment on the sensitivity, specificity, simplicity, and reliability of each endpoint that can be determined from the data that are currently available. For the purpose of this discussion, type-specificity refers to whether or not the neurobehavioral assays in question produce qualitatively different results for different pyrethroid compounds. Below, a critical analysis of the neurobehavioral data for pyrethroids is presented that identifies advantages and limitations of the examined endpoints for investigating pyrethroid-mediated neurotoxicity. In addition, comments on whether or not the results demonstrate a common adverse outcome throughout the pyrethroids that have been studied and the adequacy of the studied endpoints for use in a risk assessment process are provided.

Measurements of motor activity have several advantages for use in the assessment of pyrethroid neurotoxicity, including high sensitivity, simple testing procedures, and reproducibility across studies $[145,33,35,111,187,189]$ as well as automated collection of data that precludes observational bias. The available data indicate that all pyrethroids decrease motor activity. Thus, this neurobehavioral effect does not provide information on potential type-specific mechanisms of pyrethroid neurotoxicity. Similar to motor activity, data from rotarod- and righting-reflex tests indicate that a common adverse effect is observed across the examined pyrethroids (i.e. decreased motor coordination). Likewise, a common adverse effect is observed across different pyrethroids in schedule-controlled operant behavior (i.e. decrease in responding rates; see Table 6). For the latter two functional domains, doseresponse information using a larger variety of pyrethroids needs to be examined under standardized laboratory conditions to determine if these assays provide an appreciable increase in sensitivity over more extensively characterized endpoints like motor activity. A potential caveat of using rotarod and operant 
Table 7

Critical analysis of the toxicological data accumulated using a variety of endpoints after acute exposure to pyrethroids in rat

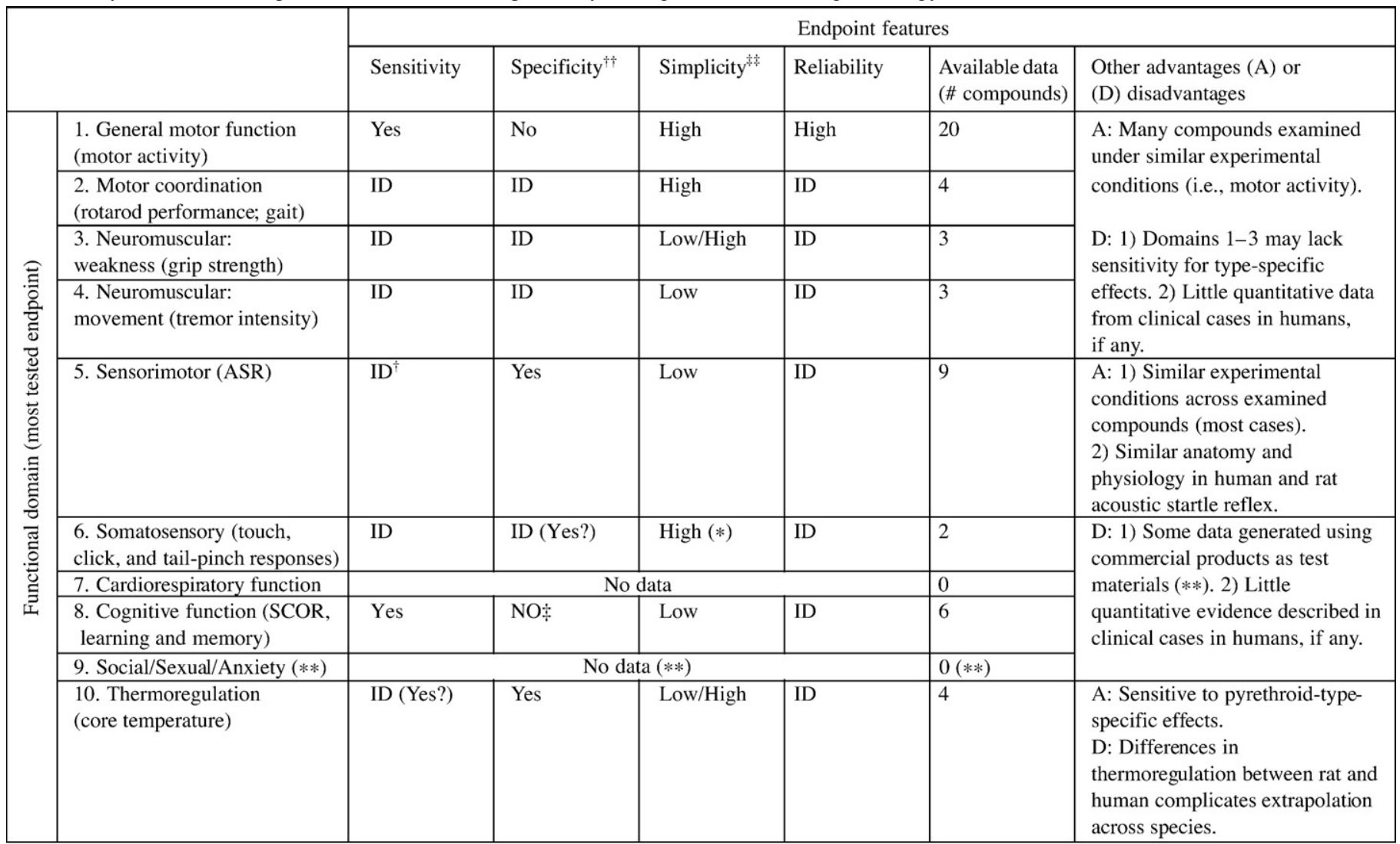

Peer-reviewed studies using technical grade compounds (most cases) and acute oral or i.p. exposures were considered. This table allows for identification of large gaps in the neurotoxicological knowledge base for pyrethroids in mammals. ${ }^{\dagger}$ Few doses examined per compound, precluding a final ascertainment; sensitivity would seem to be, at most, similar to that observed using motor activity as endpoint $[33,35,187] .{ }^{\dagger \dagger}$ Specificity stands for availability of data indicating more than one type of action of pyrethroids on this endpoint depending on structure. ${ }^{*}$ Based on schedule-controlled operant responding (SCOR) assessments (most used endpoint for this domain); limited evidence showing some divergence between permethrin and cypermethrin effects on operant responding [132] (see also Table 6). ${ }^{*}$ Simplicity refers automatization and easy execution of experimental procedures. *Provided that well-trained experimenters conduct the tests. **The little data available was obtained using commercial formulations including trade secret unknowns. "Low/High" means that the simplicity of the endpoint depends on the device and protocol used. ASR $=$ acoustic-evoked startle response. $\mathrm{ID}=$ insufficient data.

conditioning is the requirement for habituation or training of the test subjects prior to implementation of the test session/s, respectively.

The acoustic startle response (ASR) assay is the most extensively characterized neurobehavioral domain that is sensitive to pyrethroids and shows divergent effects between pyrethroid compounds (see Table 5). Note that the disparate changes in startle response latency, sensitization, and amplitude are evident in the "low" effective dose range and generally coincide with the T-(Type I)/CS-(Type II)/TS-(Type I/II) nomenclature with few exceptions. Like motor activity, the ASR assay does not require any pre-test session, and collection of data is automated and free of observational bias. An additional advantage is that the neural substrates that mediate the acoustically evoked startle reflex are known and comparable in rats and humans [31]. Data from less extensively characterized fore-/hindlimb grip strength assays (neuromuscular domain) and other FOB-protocol tests also indicate that they may be used to distinguish pyrethroid type-specific effects. Some of the FOB-related endpoints (e.g., click response, gait abnormality, and involuntary motor movements) have demon- strated acceptable sensitivity for pyrethroid neurotoxicity $[111,81,189]$. However, generalizations on their specificity for pyrethroid syndromes will await assessment of a larger number of Type I and Type II pyrethroids.

Other in vivo endpoints not described in this review, and for which little quantitative data are available, may be useful in risk analysis of pyrethroids. For instance, a thermoregulatory response is elicited in rats following pyrethroid exposure [111]. The use of telemetry for measuring core temperature $[72,43]$ in freely moving animals could be a potentially useful endpoint for assessing pyrethroid toxicity and partitioning of these compounds into separate categories. In order to determine the utility of this endpoint, single compound and mixture studies are currently being conducted using acute, oral exposures to permethrin, bifenthrin, cypermethrin, deltamethrin, and $\beta$-cyfluthrin (Wolansky et al., in progress). Preliminary results suggest 1 ) that the thermoregulatory response is affected differently by $\mathrm{T}$ - and CS-syndrome pyrethroids (i.e., permethrin and bifenthrin produce hyperthermia while deltamethrin, cypermethrin and $\beta$-cyfluthrin produce hypothermia);2) that loweffective levels of CS-compounds may induce mild effects in the 
opposite direction from what is expected after exposure to higher doses (i.e. a biphasic response); and 3) that the sensitivity of telemetered core temperature in the home cage is comparable to that of motor activity in a figure-eight maze $[33,35,36,111,187,190]$.

The selection and use of neurobehavioral data in the context of risk assessment is a contentious subject [95]. It is obvious from gross observations of pyrethroid poisoning that at least two different types of syndromes are observed in rodents at "high" doses. However, at "lower" effective doses, where gross signs of poisoning are not observed, some toxicological effects of pyrethroids are remarkably consistent across compounds (e.g., decrease of motor activity and operant response rates) while others still seem to suggest divergent toxicological profiles for different compounds (i.e., acoustic startle response). One of the quandaries that regulatory agencies face is how to interpret this complex body of data, where even within the low-effective dose range there is at least one endpoint showing disparate adverse effects with different compounds. Use of endpoints that can distinguish between the different types of pyrethroid poisoning syndromes, but are not the most sensitive endpoints available, may result in a decreased distance between allowed environmental application levels and toxicity thresholds in humans. On the other hand, using sensitive endpoints that do not separate compounds into mechanistic subcategories may result in incorrect estimates of risk associated with heterogeneous mixtures of pyrethroids with truly different toxicological pathways. In conclusion, the knowledge base summarized above indicates that one single neurobehavioral domain (or endpoint) is insufficient to ascertain the toxicological heterogeneity within the pyrethroid class. Consideration of a battery of neurobehavioral endpoints is needed to construct an accurate profile of pyrethroid toxicity for use in making wellinformed decisions for regulating pyrethroid insecticides.

\section{Conflict of interest}

The authors M.J.W. and J.A.H. state that there are no conflicts of interest applicable for this work.

\section{Acknowledgements}

The authors want to express their gratitude to Drs. M. Hornychová, J. Bloomquist, C.T. Chopde, R. MacPhail, T. Yamada, D. Gammon, M.R. Durnam, and U. Heudorf for graciously providing information on their pyrethroid studies during the data compilation process. We also thank Drs. R. MacPhail, L. Sheets, Phil Bushnell, and Ram Ramabhadran for their comments on an earlier version of this paper and Dr. K. Crofton for his highly valued guidance during the construction of this work. This work was performed while M.J.W. held a National Research Council Research Associateship Award at the National Health and Environmental Research Laboratory, US Environmental Protection Agency.

\section{Appendix A. Supplementary data}

Supplementary data associated with this article can be found, in the online version, at doi:10.1016/j.ntt.2007.10.005.

\section{References}

[1] C.O. Abernathy, J.E. Casida, Pyrethroid insecticides: esterase cleavage in relation to selective toxicity, Science 179 (1973) 1235-1236.

[2] D. Abbud Righi, J. Palermo-Neto, Behavioral effects of type II pyrethroid cyhalothrin in rats, Toxicol. Appl. Pharmacol. 191 (2) (2003) 167-176.

[3] M.B. Abou-Donia, L.B. Goldstein, K.H. Jones, A.A. Abdel-Rahman, T.V. Damodaran, A.M. Dechkovskaia, S.L. Bullman, B.E. Amir, W.A. Khan, Locomotor and sensorimotor performance deficit in rats following exposure to pyridostigmine bromide, DEET, and permethrin alone and in combination, Toxicol. Sci. 60 (2001) 305-314.

[4] Alanwood (Compendium of Pesticide Names), Cypermethrin, $\alpha$-cypermethrin, $\beta$-cypermethrin, $\theta$-cypermethrin, and $\zeta$-cypermethrin. Addendum \#2online:, www.alanwood.net/pesticides/index_rev_frame.html, 1983.

[5] W.N. Aldridge, An assessment of the toxicological properties of pyrethroids and their neurotoxicity, Crit. Rev. Toxicol. 21 (2) (1990) 89-104.

[6] R.A. Alzogaray, A. Fontan, E.N. Zerba, Evaluation of hyperactivity produced by pyrethroid treatment on third instar nymphs of Triatoma infestans (Hemiptera: Reduviidae), Arch. Insect Biochem. Physiol. 35 (3) (1997) 323-333.

[7] E.L. Amweg, D.P. Weston, N.M. Ureda, Use and toxicity of pyrethroid pesticides in the central valley, California USA, Environ. Toxicol. Chem. 24 (2005) 966-972.

[8] A. Anadón, M.R. Martínez-Larrañaga, M.L. Fernández-Cruz, M.J. Díaz, M.C. Fernández, M.A. Martínez, Toxicokinetics of deltamethrin and its 4'-HO metabolite in the rat, Toxicol. Appl. Pharmacol. 141 (1) (1996) $8-16$.

[9] A. Anadón, M. Martínez, M.A. Martínez, M.J. Díaz, M.R. MartínezLarrañaga, Toxicokinetics of lambda-cyhalothrin in rats, Toxicol. Lett. 165 (1) (2006) 47-56.

[10] S.S. Anand, K-B. Kim, S. Padilla, S. Muralidhara, H.J. Kim, J.W. Fisher, J.V. Bruckner, Ontogeny of hepatic and plasma metabolism of deltamethrin in vitro: role in age-dependent acute neurotoxicity, Drug Metab. Dispos. 34 (3) (2006) 389-397.

[11] E. Baatrup, M. Bayley, Effects of the pyrethroid insecticide cypermethrin on the locomotor activity of the wolf spider Pardosa amentata: quantitative analysis employing computer-automated video tracking, Ecotoxicol. Environ. Saf. 26 (1993) 138-152.

[12] P. Barry Ryan, T.A. Burke, E.A. Cohen Hubal, J.J. Cura, T.E. McKone, Using biomarkers to inform cumulative risk assessment, Environ. Health Perspect. 115 (2007) 833-840.

[13] M. Benoit, P. Bruneau, C. Meinard, Comparative activity of tralomethrin and deltamethrin on Periplaneta americana, Pestic. Biochem. Physiol. 26 (1986) 284-291.

[14] A.S. Bloom, C.G. Staatz, T. Dieringer, Pyrethroid effects on operant responding and feeding, Neurobehav. Toxicol. Teratol. 5 (1983) 321-324.

[15] S.P. Bradbury, J.R. Coats, Comparative toxicology of the pyrethroid insecticides, Rev. Environ. Contam. Toxicol. 108 (1989) 133-177.

[16] M.E. Brodie, W.N. Aldridge, Elevated cerebellar cyclic GMP levels during the deltamethrin-induced motor syndrome, Neurobehav. Toxicol. Teratol. 4 (1) (1982) 109-113.

[17] S.A. Burr, D.E. Ray, Structure-activity and interaction effects of 14 different pyrethroids on voltage-gated chloride ion channels, Toxicol. Sci. 77 (2004) 341-346.

[18] S.Z. Cagen, L.A. Malley, C.M. Parker, T.H. Gardiner, G.A. Van Gelder, V.A. Jud, Pyrethroid-mediated skin sensory stimulation characterized by a new behavioral paradigm, Toxicol. Appl. Pharmacol. 76 (2) (1984) 270-279.

[19] California Department of Pesticide Regulation (CDPR). Pesticide use stats. Government of California (http://www.cdpr.ca.gov/docs/pur/purmain.htm; last accesed June 2007).

[20] J.E. Casida, Pyrethrum flowers and pyrethroid insecticides, Environ. Health Perspect. 34 (1980) 189-202.

[21] J.E. Casida, G.B. Quistad, Golden age of insecticide research: past, present, or future? Annu. Rev. Entomol. 43 (1998) 1-16.

[22] J.E. Casida, K. Ueda, L.C. Gaughan, L.T. Jao, D.M. Soderlund, Structure-biodegradability relationships in pyrethroid insecticides, Arch. Environ. Contam. Toxicol. 3 (4) (1975/1976) 491-500. 
[23] CDC, Center for Disease Control and Prevention, Toxicological profile for pyrethrins and pyrethroids, Agency for Toxic Substances and Disease Registry, US Department of Health and Human Services, Atlanta, Sept 2003.

[24] CDC, Center for Disease Control and Prevention, Third national report on human exposure to environmental chemicals, Department of Health and Human Services. National Center for Environmental Health, 2005, http:// www.cdc.gov/exposurereport/3rd/pdf/thirdreport.pdf.

[25] P.H. Chanh, C. Navarro-Delmasurre, A.P.H. Chanh, S.L. Cheav, F. Ziade, F. Samaha, Pharmacological effects of deltamethrin on the central nervous system, Arzneim.-Forsch. (Drug Res.) 34 (I) (2) (1984) 175-181.

[26] P.H. Chanh, C. Navarro-Delmasurre, A.P.H. Chanh, P. Clavel, G. Van Haverbeke, S.L. Cheav, Toxicological studies of deltamethrin, Int. J. Tissue React. VI (2) (1984) 127-133.

[27] J.S. Choi, D.M. Soderlund, Structure-activity relationships for the action of 11 pyrethroid insecticides on rat Na v 1.8 sodium channels expressed in Xenopus oocytes, Toxicol. Appl. Pharmacol. 211 (3) (2006) 233-244.

[28] J.R. Coats, Mechanisms of toxic action and structure-activity relationships for organochlorine and synthetic pyrethroid insecticides, Environ. Health Perspect. 87 (1990) 255-262.

[29] J.E. Cremer, The influence in mammals of the pyrethroid insecticides, Dev. Toxicol. Environ. Sci. 11 (1983) 61-72.

[30] J.E. Cremer, M.P. Seville, Comparative effects of two pyrethroids, deltamethrin and cismethrin, on plasma catecholamines and on blood glucose and lactate, Toxicol. Appl. Pharmacol. 66 (1982) 124-133.

[31] K.M. Crofton, Reflex modification and the detection of toxicant-induced auditory dysfunction, Neurotoxicol. Teratol. 12 (5) (1990) 461-468.

[32] K.M. Crofton, R.C. MacPhail, Reliability of motor assessments, in: K.P. Ossenkopp, M. Kavaliers, P.R. Sanberg (Eds.), Measuring Movement and Locomotion: from Invertebrates to Humans, R.G. Landes Company, 1996, pp. 227-252, Chapter 12.

[33] K.M. Crofton, L.W. Reiter, Effects of two pyrethroids on motor activity and the acoustic startle response in the rat, Toxicol. Appl. Pharmacol. 75 (1984) 318-328.

[34] K.M. Crofton, L.W. Reiter, Pyrethroid insecticides and the gammaaminobutyric acid A receptor complex: motor activity and the acoustic startle response in the rat, J. Pharmacol. Exp. Ther. 243 (3) (1987) 946-954.

[35] K.M. Crofton, L.W. Reiter, The effects of type I and II pyrethroids on motor activity and the acoustic startle response in the rat, Fundam. Appl. Toxicol. 10 (1988) 624-634.

[36] K.M. Crofton, L.S. Kehn, M.E. Gilbert, Vehicle and route dependent effects of a pyrethroid insecticide, deltamethrin, on motor function in the rat, Neurotoxicol. Teratol. 17 (4) (1995) 489-495.

[37] K.M. Crofton, J.L. Howard, V.C. Moser, M.W. Gill, L.W. Reiter, H.A Tilson, R.C. MacPhail, Inter-laboratory comparisons of motor activity experiments: implications for neurotoxicological risk assessments, Neurotoxicol. Teratol. 13 (6) (1991) 599-609.

[38] M. Dayal, D. Parmar, A. Dhawan, M. Ali, U.N. Dwivedi, P.K. Seth, Effect of pretreatment of cytochrome P450 (P450) modifiers on neurobehavioral toxicity induced by deltamethrin, Food Chem. Toxicol. 41 (2003) 431-437.

[39] S.F. de Boer, J. van der Gugten, J.L. Slangen, T.H. Hijzen, Changes in plasma corticosterone and catecholamine induced by low doses of deltamethrin in rats, Toxicology 49 (1988) 263-270.

[40] DEFRA, Department for Environment, Food, and Rural Affairs, Pesticide usage statistics. Central Science Laboratory and Scottish Agricultural Science Agency, UK, http://pusstats.csl.gov.uk/index.cfm, 2006.

[41] H. De Souza Spinosa, Y.M. Silva, A.A. Nicolau, M.M. Bernardi, A. Lucisano, Possible anxiogenic effects of fenvalerate, a type II pyrethroid pesticide, in rats, Physiol. Behav. 67 (1999) 611-615.

[42] V.G. Dethier, L.B. Barton-Browne, C.N. Smith, The designation of chemicals in terms of the responses they elicit from insects, J. Econ. Entomol. 53 (1960) 134-136.

[43] A.M. Deveney, A. Kjellstrom, T. Forsberg, D.M. Jackson, A pharmacological validation of radiotelemetry in conscious, freely moving rats, J. Pharmacol. Toxicol. Methods 40 (2) (1998) 71-79.

[44] D.C. Dorman, V.R. Beasley, Neurotoxicology of pyrethrin and pyrethroid insecticides, Vet. Hum. Toxicol. 33 (3) (1991) 238-243.
[45] D.C. Dorman, W.B. Buck, H.L. Trammel, R.D. Jones, V.R. Beasley, Fenvalerate $/ N, N$-diethyl- $m$-toluamide (DEET) toxicosis in two cats, J. Am. Vet. Med. Assoc. 196 (1) (1990) 100-102.

[46] EC-HCPDG, European Commission, Health \& Consumer Protection Directorate-General, Directorate E1: Plant Health. Beta-Cyfluthrin, Review Report \# 6841/VI/97-final, , 12-2-2002.

[47] R. Edwards, P. Millburn, D.H. Hutson, Comparative toxicity of ciscypermethrin in rainbow trout, frog, mouse, and quail, Toxicol. Appl. Pharmacol. 84 (1986) 512-522.

[48] M. Elliott, N.F. Janes, Synthetic Pyrethroids: a New Class of Insecticide, Chem. Soc. Rev. (London), 7 (4), 1978, pp. 470-505.

[49] EMEA, European Agency for the Evaluation of Medicinal Products, Taufluvalinate EMEA/MRL/021-REV1/95, Revised Summary Report, Committee for Veterinary Medicinal Products, , 1995.

[50] EMEA, European Agency for the Evaluation of Medicinal Products, Cypermethrin. Summary Report. EMEA/MRL/403/98-FINAL, , 1998.

[51] EMEA, European Agency for the Evaluation of Medicinal Products, EMEA/MRL/362/98-FINAL, Chrysanthemi cinerariifolii flos and pyrethrum extract, summary report, , Febr. 1998.

[52] EMEA, European Agency for the Evaluation of Medicinal Products, $\alpha$ Cypermethrin EMEA/MRL/879/03-FINAL, Summary Report (3), June 2003.

[53] FAN, Fluoride Action Network. Transfluthrin. In: Flurorinated and fluoride pesticides. CAS No. 118712-89-3 (taken from: www.fluoridealert.org/ pesticides/epage.transfluthrin.htm). Evaluation on: Transfluthrin use as a public hygiene insecticide, UK Health and Safety Executive, Biocides \& Pesticides Assessment Unit, Magdalen House, Stanley Precinct, Bootle, Merseyside, UK, September 1997 (full document available from: Department for Environment, Food and Rural Affairs, Pesticides Safety Directorate, York, UK).

[54] FDA, US Food and Drug Administration, Total diet study [summary of residues found, ordered by pesticide]: market baskets 91-3-01-4, http:// www.cfsan.fda.gov/ comm/tds-res.html, June 2003.

[55] M.G. Ford, N.E. Hoare, B.D. Hudson, T.G. Nevell, L. Banting, QSAR studies of the pyrethroid insecticides. Part 3. A putative pharmacophore derived using methodology based on molecular dynamics and hierarchical cluster analysis, J. Mol. Graph. Model 21 (1) (2002) 29-36.

[56] FQPA. The Food Quality Protection Act of 1996. Public Law 104-170.

[57] G.S. Fraenkel, D.L. Gunn, The Orientation of Animals, Kineses, Taxes, and Compass Reactions, Dover, New York, 1961.

[58] Freedonia, Pesticides, Freedonia Industry Study, vol. 1523, The Freedonia Market Research Group, Inc., Cleveland, OH, 2006.

[59] Y. Fujitani, Chemistry and pharmacology of insect powder, Arch. Exp. Pathol. Pharmacol. 61 (1909) 47-75.

[60] D.W. Gammon, Correlations between in vivo and in vitro mechanisms of pyrethroid insecticide action, Fundam. Appl. Toxicol. 5 (1) (1985) 9-23.

[61] D.W. Gammon, M.A. Brown, J.E. Casida, Two classes of pyrethroid action in the cockroach, Pestic. Biochem. Physiol. 15 (1981) 181-191.

[62] D.W. Gammon, L.J. Lawrence, J.E. Casida, Pyrethroid toxicology: protective effects of diazepam and phenobarbital in the mouse and the cockroach, Toxicol. Appl. Pharmacol. 66 (1982) 290-296.

[63] L.C. Gaughan, T. Unai, J.E. Casida, Permethrin metabolism in rats, J. Agric. Food Chem. 25 (1977) 9-17.

[64] M.E. Gilbert, S.K. Acheson, C.M. Mack, K.M. Crofton, An examination of the proconvulsant actions of pyrethroid insecticides using pentylenetetrazol and amygdala kindling seizure models, Neurotoxicology 11 (1) (1990) 73-86.

[65] K. Ginsburg, T. Narahashi, Time course and temperature dependence of allethrin modulation of sodium channels in rat dorsal root ganglion cells, Brain Res. 847 (1) (1999) 38-49.

[66] A.H. Glickman, J.E. Casida, Species and structural variations affecting pyrethroid neurotoxicity, Neurobehav. Toxicol. Teratol. 4 (1982) 793-799.

[67] A.H. Glickman, S.D. Weitman, J.J. Lech, Differential toxicity of transpermethrin in rainbow trout and mice: I. Role of biotransformation, Toxicol. Appl. Pharmacol. 66 (2) (1982) 153-161.

[68] R. Glomot, Toxicity of deltamethrin to higher vertebrates, Deltamethrin (Monograph), Roussel-Uclaf Research Centre, France, 1982, pp. 109-136, Chapter 4. 
[69] J.R. Glowa, Acute and subacute effects of deltamethrin and chlordimeform on schedule-controlled responding in the mouse, Neurobehav. Toxicol. Teratol. 8 (1986) 97-102.

[70] S.J. Godin, E.J. Scollon, M.F. Hughes, P.M. Potter, M.J. DeVito, M.K. Ross, Species differences in the in vitro metabolism of deltamethrin and esfenvalerate: differential oxidative and hydrolytic metabolism by humans and rats, Drug Metab. Dispos. 34 (10) (2006) 1764-1771.

[71] C.J. Gordon, Effect of cage bedding on temperature regulation and metabolism of group-housed female mice, Comp. Med. 54 (1) (2004) 50-55.

[72] C.J. Gordon, Temperature and Toxicology: an Integrative, Comparative, and Environmental Approach, CRC Press, Boca Raton, Florida, 2005.

[73] A.J. Gray, Pyrethroid structure-toxicity relationships in mammals, Neurotoxicology 6 (2) (1985) 127-138.

[74] A.J. Gray, D.M. Soderlund, Mammalian toxicology of pyrethroids, in: D.H. Hutson, T.R. Roberts (Eds.), Insecticides, John Wiley \& Sons Ltd., New York, 1985, pp. 193-248.

[75] A.J. Gray, T.A. Connors, H. Hoellinger, H-N. Nguyen, The relationship between the pharmacokinetics of intravenous cismethrin and biomesthrin and their mammalian toxicity, Pestic. Biochem. Physiol. 13 (3) (1980) 281-293.

[76] K. Greulich, S. Pflugmacher, Uptake and effects on detoxification enzymes of cypermethrin in embryos and tadpoles of amphibians, Arch. Environ. Contam. Toxicol. 47 (2004) 489-495.

[77] D.W. Herr, J-S. Hong, P. Chen, H. Tilson, G.J. Harry, Pharmacological modification of DDT-induced tremor and hyperthermia in rats: distributional factors, Psychopharmacol. 89 (1986) 278-283.

[78] T.H. Hijzen, J.L. Slangen, Effects of type I and type II pyrethroids on the startle response in rats, Toxicol. Lett. 40 (2) (1988) 141-152.

[79] T.H. Hijzen, R. De Beun, J.L. Slangen, Effects of pyrethroids on the acoustic startle reflex in the rat, Toxicology 49 (2-3) (1988) 271-276.

[80] J.L. Holton, C.C. Nolan, S.A. Burr, D.E. Ray, J.B. Cavanagh, Increasing or decreasing nervous activity modulates the severity of the glio-vascular lesions of 1,3-dinitrobenzene in the rat: effects of the tremorgenic pyrethroid, bifenthrin, and of anaesthesia, Acta Neuropathol. 93 (1997) 159-165.

[81] M. Hornychová, E. Frantik, J. Kubat, J. Formanek, Neurotoxicity profile of supermethrin, a new pyrethroid insecticide, Cent. Eur. J. Public Health 3 (4) (1995) 210-218.

[82] J.B. Hoy, J.A. Cornell, J.L. Karlix, C.J. Schmidt, I.R. Tebbet, F. van Haaren, Interactions of pyridostigmine bromide, DEET and permethrin after locomotor behavior of rats, Vet. Hum. Toxicol. 42 (2) (2000) 65-71.

[83] P.M. Hudson, H.A. Tilson, P.H. Chen, J.S. Hong, Neurobehavioral effects of permethrin are associated with alterations in regional levels of biogenic amine metabolites and amino acid neurotransmitters, Neurotoxicology 7 (1) (1986) 143-154

[84] G.M. Hughes, P. Biro, Swimming performance of rainbow trout following exposure and recovery from the pyrethroid $S$-deltamethrin, Acta Biol. Hung. 44 (2-3) (1993) 231-241.

[85] R. Husain, R. Husain, V.M. Adhami, P.K. Seth, Behavioral, neurochemical, and neuromorphological effects of deltamethrin in adult rats, J. Toxicol. Environ. Health 48 (5) (1996) 515-526.

[86] P.J.C.M. Janssen, P.H. Arentzen, E.M. den Tonkelaar, Ethofenprox: Pesticide Residues in Food, Part. II, Toxicology (First Draft), National Institute of Public Health and Environmental Protection, International Programme on Chemical Safety Poisons Information (IPCS-INCHEM), Bilthoven, Netherlands, 1993.

[87] R. Julien, G. Adamkiewicz, J.I. Levy, D. Bennett, M. Nishioka, J.D. Spengler, Pesticide Loadings of Select Organophosphate and Pyrethroid Pesticides in Urban Public Housing, , 2007.

[88] J. Kamei, M. Sasaki, K. Sushida, K. Morita, S. Tanaka, Nociception and allodynia/hyperalgesia induced by intrathecal administration of fenvalerate, Jpn. J. Pharmacol. 86 (3) (2001) 336-341.

[89] J. Kamei, A. Saitoh, N. Tamura, Effect of mexiletine on fenvalerateinduced nociceptive response in diabetic mice, J. Pharmacol. Sci. 96 (3) (2004) 280-285.

[90] D.J. Karen, W. Li, P.R. Harp, JS. Gillette, J.R. Bloomquist, Striatal dopaminergic pathways as a target for the insecticides permethrin and chlorpyrifos, Neurotoxicology 22 (2001) 811-817.
[91] Y. Katsuda, Development of and future prospects for pyrethroid chemistry, Pestic. Sci. 55 (1999) 775-782.

[92] R. Kavlock, N. Chernoff, R. Baron, R. Linder, E. Rogers, B. Carver, Toxicity studies with decamethrin, a synthetic pyrethroid insecticide, J. Environ. Pathol. Toxicol. 2 (1979) 751-765.

[93] B.P.S. Khambay, Pyrethroid insecticides. Royal Society of Chemistry (UK), Pestic. Outlook (April 2002) 49-54.

[94] J.H. Kolaczinski, C.F. Curtis, Chronic illness as a result of low-level exposure to synthetic pyrethroid insecticides: a review of the debate, Food Chem. Toxicol. 42 (2004) 697-706.

[95] B. Kulig, E. Alleva, G. Bignami, J. Cohn, D. Cory-Slechta, V. Landa, J. O'Donoghue, D. Peakall, Animal behavioral methods in neurotoxicity assessment: SGOMSEC joint report, Environ. Health Perspect. 104 (Suppl. 2) (1996) 193-204 see also: http://www.ehponline.org/members/ 1996/Suppl-2/bkulig.html.

[96] N. Kurihara, J. Miyamoto, G.D. Paulson, B. Zeeh, M.W. Skidmore, R.M. Hollingworth, H.A. Kuiper, IUPAC reports on pesticides (37), chirality in synthetic agrochemicals: bioactivity and safety consideration (technical report), Pure Appl. Chem. 69 (9) (1997) 2007-2025.

[97] D.A. Laskowski, Physical and chemical properties of pyrethroids, Rev. Environ. Contam. Toxicol. 174 (2002) 49-170.

[98] L. Lawrence, J.E. Casida, Pyrethroid toxicology: mouse intra-cerebral structure-toxicity relationships, Pestic. Biochem. Physiol. 18 (1982) 9-14.

[99] J. Legath, J. Neuschl, P. Kacmar, J. Poracova, E. Dudrikova, H. Hlynarcikova, G. Kovac, P. Javorsky, Clinical signs and mechanisms of supermethrin intoxication in sheep, Vet. Hum. Toxicol. 34 (5) (1992) 453-455.

[100] G. Leng, W. Gries, S. Selim, Biomarker of pyrethrum exposure, Toxicol. Lett. 162 (2-3) (2006) 195-201.

[101] I.V. Lepeshkin, I.I. Mokov, S.V. Murashko, IuS. Kagan, [The effect of solvents and emulsifiers on the toxicity of synthetic pyrethroids], Lik Sprava (Likars'ka sprava/Ministerstvo okhorony zdorov'ia Ukraïny), 10, 1992, pp. 67-69.

[102] W. Liu, J. Gan, D. Schlenk, W.A. Jury, Enantioselectivity in environmental safety of current chiral insecticides, PNAS (USA) 102 (3) (2005) 701-706.

[103] R.C. MacPhail, W.A. Gordon, M.A. Johnston, Behavioral effects of decamethrin, Fed. Proc. 40 (1981) 678.

[104] L.A. Malley, S.Z. Cagen, C.S. Parker, T.H. Gardiner, G.A. Van Gelder, G.P. Rose, Effect of vitamin E and other amelioratory agents on the fenvaleratemediated skin sensation, Toxicol. Lett. 29 (1) (1985) 51-58.

[105] S.N. Mandhane, C.T. Chopde, Neurobehavioral effects of low-leve fenvalerate exposure in mice, Indian J. Exp. Biol. 35 (6) (1997) 623-627.

[106] S. Manna, D. Bhattacharyya, T.K. Mandal, S. Das, Repeated dose toxicity of alpha-cypermethrin, J. Vet. Sci. 5 (3) (2004) 241-245.

[107] S. Manna, D. Bhattacharyya, T.K. Mandal, S. Dey, Neuropharmacological effects of alpha-cypermethrin in rats, Indian J. Pharmacol. 37 (1) (2005) 18-20.

[108] S. Manna, D. Bhattacharyya, T.K. Mandal, S. Dey, Neuropharmacological effects of deltamethrin in rats, J. Vet. Sci. 7 (2) (2006) 133-136.

[109] J. Marshall Clark, M.W. Brooks, Neurotoxicology of pyrethroids: single or multiple mechanisms of action? Environ. Toxicol. Chem. 8 (1989) 361-372.

[110] J.P. Maurissen, J.L. Mattsson, Critical assessment of motor activity as a screen for neurotoxicity, Toxicol. Ind. Health 5 (2) (1989) 195-202.

[111] K.L. McDaniel, V.C. Moser, Utility of a neurobehavioral screening battery for differentiating the effects of two pyrethroids, permethrin and cypermethrin, Neurotoxicology 15 (1993) 71-83.

[112] D.B. McGregor, Permethrin, Joint Meeting, FAO Panel of Experts on Pesticide Residues in Food and the Environment, and WHO Core Assessment Group, Rome, 1999.

[113] D.B. McGregor, Esfenvalerate, Pesticide Residues in Food. Joint FAO/ WHO Meeting on Pesticide Residues, 2002 www.inchem.org/documents/ jmpr/jmpmono/2002pr04.htm.

[114] L. Metker, R.A. Angerhofer, C.R. Pope, K.C. Swentzel, Toxicological evaluation of 3-(phenoxyphenyl) methyl $( \pm)$-cis, trans-3-(2,2-dichloroethenyl)-2,2-dimethylciclopropanecarboxylate (permethrin). Sudy \#510831-78, Department of the Army, US Environmental Hygiene Agency, Aberdeen Proving Ground, Maryland, December 11977. 
[115] T.A. Miller, M.E. Adams, Modes of action of pyrethroids, in: J.R. Coats (Ed.), Insecticide Mode of Action, Academy Press, 1982, pp. 3-27.

[116] A. Mirfazaelian, K.B. Kim, S.S. Anand, H.J. Kim, R. Tornero-Velez, J.V. Bruckner, Development of a physiologically based pharmacokinetic model for deltamethrin in the adult male Sprague-Dawley rat, Toxicol. Sci. 93 (2) (2006) 432-442.

[117] J.A. Mitchell, M.C. Wilson, M.J. Kallman, Behavioral effects of pydrin and ambush in male mice, Neurotoxicol. Teratol. 10 (1988) 113-119.

[118] J. Miyamoto, Degradation, metabolism and toxicity of synthetic pyrethroids, Environ. Health Perspect. 14 (1976) 15-28.

[119] K.D. Moran, Sources of Pyrethroids in Urban Run-off. UP3 Project, State of the Estuary Conference, TDC Environmental LLC, California, October 4-6 2005.

[120] H. Motomura, T. Narahashi, Interaction of tetramethrin and deltamethrin at the single sodium channel in rat hippocampal neurons, Neurotoxicology 22 (2001) 329-339.

[121] M.E. Mount, G. Moller, J. Cook, D.M. Holstege, E.R. Richardson, A. Ardans, Clinical illness associated with a commercial tick and flea product in dogs and cats, Vet. Hum. Toxicol. 33 (1) (1991) 19-27.

[122] T. Narahashi, Neuroreceptors and ion channels as the basis for drug action: past, present, and future, J. Pharmacol. Exp. Ther. 294 (1) (2000) $1-26$.

[123] T. Narahashi, K.S. Ginsburg, K. Nagata, J.H. Song, H. Tatebayashi, Ion channels as targets for insecticides, Neurotoxicology 19 (4-5) (1998) 581-590.

[124] NAS, US National Academy of Sciences, Pharmacokinetics of permethrin. In: health effects of permethrin-impregnated army battledress uniforms, Commission of Life Sciences, National Academies Press, Washington, DC, 1994, pp. 27-42, Chapter 3, (taken from electronic version, http://www.nap.edu/books/NI000104/html/index.html).

[125] K. Naumann, Synthetic pyrethroid insecticides: structures and properties, Chemistry of Plant Protection, Vol. 4, Springer Verlag, Berlin, 1990.

[126] K. Naumann, Research into fluorinated pyrethroid alcohols: an episode in the history of pyrethroid discovery, Pestic. Sci. 52 (1998) 3-20.

[127] M. Nishimura, N. Obana, O. Yagasaki, I. Yanagiya, Involvement of adrenergic and serotoninergic nervous mechanisms in allethrin-induced tremors in mice, J. Toxicol. Sci. 9 (1984) 131-142.

[128] OECD, Organisation for Economic Co-operation and Development, Series on testing and assessment: guidance document for neurotoxicity testing, Environment Directorate. Joint Meeting of the Chemicals Committee and the Working Party on Chemicals, Pesticides and Biotechnology. ENV/JM/ MONO (2004) 25, 26-Nov-2004, available at: www.oecd.org/ehs/.

[129] A.O. O'Reilly, B.P. Khambay, M.S. Williamson, L.M. Field, B.A. Wallace, T.G. Davies, Modelling insecticide-binding sites in the voltagegated sodium channel, Biochem. J. 396 (2) (2006) 255-263.

[130] C.M. Parker, V.J. Piccirillo, S.L. Kurtz, F.M. Garner, T.H. Gardiner, G.A. Van Gelder, Six month feeding study of fenvalerate in dogs, Fundam. Appl. Toxicol. 4 (1984) 577-586.

[131] C.M. Parker, J.R. Albert, G.A. Van Gelder, D.R. Patterson, J.L. Taylor, Neuropharmacologic and neuropathologic effects of fenvalerate in mice and rats, Fundam. Appl. Toxicol. 5 (2) (1985) 278-286.

[132] D.B. Peele, K.M. Crofton, Pyrethroid effects on schedule-controlled behavior: time and dosage relationships, Neurotoxicol. Teratol. 9 (1987) 387-394.

[133] PMRA, Canada Pest Management Regulatory Agency, Report PRDD \#200502 (imiprothrin), www.pmra-arla.gc.ca/english/pdf/prdd/prdd2005-02-e.pdf, Aug 82005

[134] G.D. Pollak, R.M. Burger, A. Klug, Dissecting the circuitry of the auditory system, Trends Neurosci. 26 (1) (2003) 33-39.

[135] M.E.J. Pronk, G.J.A. Speijers, M.F.A. Wouters, L. Ritter, Cypermethrin and alpha-cypermethrin, Food Additive Series \#38. Toxicology Advisory Centre, National Institute of Public Health and Environmental Protection, International Programme on Chemical Safety Poisons Information, Bilthoven, Netherlands, and Canadian Network of Toxicology Centres, University of Guelph, Ontario, Canada, 1996, www.inchem.org/documents/jecfa/jecmono/v38je07.htm.

[136] G.T. Pryor, E.T. Uyeno, H.A. Tilson, C.L. Mitchell, Assessment of chemicals using a battery of neurobehavioral tests: a comparative study, Neurobehav. Toxicol. Teratol. 5 (1983) 91-117.
[137] F. Punzo, Detoxification enzymes and the effects of temperature on the toxicity of pyrethroids to the fall armyworm, Spodoptera frugiperda (Lepidoptera: Noctuidae), Comp. Biochem. Physiol. Part C: Comp. Pharmacol. 105 (2) (1993) 155-158.

[138] W.D. Ratnasooriya, S.S. Ratnayake, Y.N. Jayatunga, Effects of pyrethroid insecticide ICON ( $\lambda$-cyhalothrin) on reproductive competence of male rats, Asian J. Androl. 4 (2002) 35-41.

[139] D.E. Ray, The contrasting actions of two pyrethroids (deltamethrin and cismethrin) in the rat, Neurobehav. Toxicol. Teratol. 4 (6) (1982) 801-804.

[140] D.E. Ray, Function in neurotoxicity: index of effect and also determinant of vulnerability, Clin. Exp. Pharmacol. Physiol. 24 (11) (1997) $857-860$.

[141] D.E. Ray, J.E. Cremer, The action of decamethrin (a synthetic pyrethroid) on the rat, Pestic. Biochem. Physiol. 10 (1979) 333-340.

[142] D.E. Ray, J.R. Fry, A reassessment of the neurotoxicity of pyrethroid insecticides, Pharmacol. Ther. 111 (1) (2006) 174-193.

[143] D.E. Ray, S.A. Burr, T. Lister, The effects of combined exposure to the pyrethroids deltamethrin and $S$-bioallethrin on hippocampal inhibition and skeletal muscle hyperexcitability in rats, Toxicol. Appl. Pharmacol. 216 (2) (2006) 354-362.

[144] L.W. Reiter, R.C. MacPhail, Motor activity: a survey of methods with potential use in toxicity testing, Neurobehav. Toxicol. 1 (Suppl. 1) (1979) $53-66$.

[145] L.W. Reiter, R.C. MacPhail, P.H. Ruppert, D.A. Eckerman, Animal models of toxicity: some comparative data on the sensitivity of behavioral tests, 11th Conference on Environmental Toxicology (Nov 18-20, 1980). Report AFAMRL-TR-80-125, 1981, pp. 11-23.

[146] J. Rickard, M.E. Brodie, Correlation of blood and brain levels of the neurotoxic pyrethroid deltamethrin with the onset of symptoms in rats, Pestic. Biochem. Physiol. 23 (1985) 143-156.

[147] T.R. Roberts, D.H. Hutson, Insecticides and funguicides: pyrethroids, in: T.R. Roberts (Ed.), Metabolic Pathways of Agrochemicals, Royal Society of Chemistry, Cambridge (Great Britain), 1998, pp. 579-726, Part. 2.

[148] M.K. Ross, A. Borazjani, C.C. Edwards, P.M. Potter, Hydrolytic metabolism of pyrethroids by human and other mammalian carboxylesterases, Biochem. Pharmacol. 71 (5) (2006) 657-669.

[149] L.O. Ruzo, T. Unai, J.E. Casida, Decamethrin metabolism in rats, J. Agric. Food Chem. 26 (4) (1978) 918-925.

[150] L.O. Ruzo, J.L. Engel, J.E. Casida, Decamethrin metabolites from oxidative, hydrolytic, and conjugative reactions in mice, J. Agric. Food Chem. 27 (4) (1979) 725-731.

[151] K. Saitoh, S. Shaw, H.A. Tilson, Noradrenergic influence on the prepulse inhibition of acoustic startle, Toxicol. Lett. 34 (2-3) (1986) 209-216.

[152] R.M. Sawicki, Insecticidal activity of pyrethrum extract and its four insecticidal constituents against house fly. III. Knock-down and recovery of flies treated with pyrethrum extract with and without piperonyl butoxide, J. Sci. Food Agric. 13 (1962) 283-292.

[153] T.J. Shafer, D.A. Meyer, Effects of pyrethroids on voltage-sensitive calcium channels: a critical evaluation of strengths, weaknesses, data needs, and relationship to assessment of cumulative neurotoxicity, Toxicol. Appl. Pharmacol. 196 (2) (2004) 303-318.

[154] T.J. Shafer, D.A. Meyer, K.M. Crofton, Developmental neurotoxicity of pyrethroid insecticides: critical review and future research needs, Environ. Health Perspect. 113 (2005) 123-136.

[155] D.M. Soderlund, D.C. Knipple, The molecular biology of knockdown resistance to pyrethroid insecticides, Insect Biochem. Mol. Biol. 33 (6) (2003) 563-577.

[156] D.M. Soderlund, J.M. Clark, L.P. Sheets, L.S. Mullin, V.J. Picirillo, D. Sargent, J.T. Stevens, M.L. Weiner, Mechanisms of pyrethroid neurotoxicity: implications for cumulative risk assessment, Toxicology 171 (2002) 3-59

[157] F. Souyri, H. Hoellinguer, Neurotoxicity of pyrethrins in warm-blooded animals, Toxicol. Eur. Res. 5 (3) (1983) 103-112.

[158] J.L. Stanley, R.J. Lincoln, T.A. Brown, L.M. McDonald, G.R. Dawson, D.S. Reynolds, The mouse beam walking assay offers improved sensitivity over the mouse rotarod in determining motor coordination deficits induced by benzodiazepines, Psychopharmacol. 19 (3) (2005) 221-227. 
[159] E.A. Stein, M. Washburn, C. Walczak, A.S. Bloom, Effects of pyrethroid insecticides on operant responding maintained by food, Neurotoxicol. Teratol. 9 (1) (1987) 27-31.

[160] N.R. Swerdlow, D.L. Braff, M.A. Geyer, Cross-species studies of sensorimotor gating of the startle reflex, Ann. N.Y. Acad. Sci. 877 (1999) 202-216.

[161] J.T. Tapp, R.S. Zimmerman, P.S. D’Encarnaçao, Inter-correlational analysis of some common measures of rat activity, Psychol. Rep. 23 (1968) 1047-1050.

[162] H.A. Tilson, V.C. Moser, Comparison of screening approaches, Neurotoxicology 13 (1) (1992) 1-13.

[163] D. Tomalik-Scharte, A. Lazar, J. Meins, B. Bastian, M. Ihrig, B. Wachall, A. Jetter, I. Tantcheva-Poór, G. Mahrle, U. Fuhr, Dermal absorption of permethrin following topical administration, Eur. J. Clin. Pharmacol. 61 (5-6) (2005) 399-404.

[164] R. Tsuji, N. Isobe, H. Kawasaki, Effect of pyrethroids on pentobarbitalinduced sleeping time in relation to the chemical structure, Toxicology $106(1-3)(1996)$ 131-137.

[165] R. Tsuji, N. Isobe, H. Kawasaki, Mechanism of prolongation of pentobarbital-induced sleeping time by empethrin in mice, Toxicology 108 (3) (1996) 185-190.

[166] N.S. Tulve, P.A. Jones, M.G. Nishioka, R.C. Fortmann, C.W. Croghan, J.Y. Zhou, A. Fraser, C. Cave, W. Friedman, Pesticide measurements from the First National Environmental Health Survey of child care centers using a multi-residue GC/MS analysis method, Environ. Sci. Technol. 40 (20) (2006) 6269-6274.

[167] K. Ueda, L.C. Gaughan, J.E. Casida, Metabolism of (+)-trans- and (+)-cisresmethrin in rats, J. Agric. Food Chem. 23 (1) (1975) 106-115.

[168] USEPA, Environmental Protection Agency, Reference dose (RfD): description and use in health risk assessments, Integrated Risk Information System (IRIS). Background Document 1A, March 151993 (online: http://www.epa.gov/iris/rfd.htm).

[169] USEPA, Environmental Protection Agency, Guidelines for neurotoxicity risk assessment, Federal Register, vol. 63, \#93, 1998, pp. 26925-26954, (http://www.epa.gov/fedrgstr/EPA-TOX/1998/May/Day-14/t12303.htm).

[170] USEPA, Environmental Protection Agency, Guidance on Cumulative Risk Assessment of Pesticide Chemicals that have a Common Mechanism of Toxicity, Office of Pesticide Programs, Washington, D.C., 2002 www.epa. gov/pesticides/trac/science/cumulative_guidance.pdf.

[171] H. Vais, M.S. Williamson, A.L. Devonshire, P.N. Usherwood, The molecular interactions of pyrethroid insecticides with insect and mammalian sodium channels, Pest Manag. Sci. 57 (10) (2001) 877-888.

[172] W.M. Valentine, Pyrethrin and pyrethroid insecticides, Vet. Clin. North Am., Large Anim. Pract. 20 (1990) 375-385.

[173] J. Van den Bercken, H.P.M. Vijverberg, Interaction of pyrethroids and DDTlike compounds with the sodium channels in the nerve membrane, in: S. Matsunaka, D.H. Hutson, S.D. Murphy (Eds.), Pesticide Chemistry: Human Welfare and the Environment. In: Proceedings of the 5th International Congress of Pesticide Chemistry, Kyoto, Japan-1982, vol. 3, Mode of Action, Metabolism, and Toxicology, Pergamon Press, 1982, pp. 115-121.

[174] F. Van Haaren, B. Cody, J.B. Hoy, J.L. Karlix, C.J. Schmidt, I.R. Tebbett, D. Wielbo, The effects of pyridostigimine bromide and permethrin, alone or in combination, on response acquisition in male and female rats, Pharmacol. Biochem. Behav. 66 (4) (2000) 739-746.

[175] R.D. Verschoyle, W.N. Aldridge, Structure-activity relationships of some pyrethroids in rats, Arch. Toxicol. 45 (1980) 325-329.

[176] R.D. Verschoyle, J.M. Barnes, Toxicity of natural and synthetic pyrethrins to rats, Pestic. Biochem. Physiol. 2 (1972) 308-311.

[177] H.P.M. Vijverberg, J. Van den Bercken, Neurotoxicological effects and the mode of action of pyrethroid insecticides, Crit. Rev. Toxicol. 21 (2) (1990) 105-126.
[178] S.G. Waxman, T.R. Cummins, J. Black, S. Dib-Haij, Diverse functions and dynamic expression of neuronal sodium channels, Novartis Found. Symp. 241 (1) (2002) 34-51.

[179] D. Weinert, J. Waterhouse, Diurnally changing effects of locomotor activity in body temperature on laboratory mice, Physiol. Behav. 63 (5) (1998) 837-843.

[180] I.N.H. White, R.D. Verschoyle, M.H. Moradian, J.M. Barnes, The relationship between brain levels of cismethrin and bioresmethrin in female rats and neurotoxic effects, Pestic. Biochem. Physiol. 6 (1976) 491-500.

[181] WHO, World Health Organization, Evaluation of the toxicity of pesticide residues in food: dimethrin. FAO meeting report PL/1965/10/1. Food and Agriculture Organization (United Nations), www.inchem.org/documents/ jmpr/jmpmono/v065pr21.htm, 1965.

[182] WHO, World Health Organization, Permethrin. Environmental Health Criteria (EHC) 94, International Programme on Chemical Safety, Geneva, 1990.

[183] WHO, World Health Organization, Neurotoxicity Risk Assessment for Human Health: Principles and Approaches. IPCS-INCHEM, Environmental Health Criteria \# 223, Geneve, 2001 www.inchem.org/documents/ ehc/ehc/ehc223.htm.

[184] WHO, World Health Organization, The WHO Recommended Classification of Pesticides by Hazard. Guidelines to Classification 2004, International Programme on Chemical Safety. WHO/PCS/01.5, Geneve, 2005 http://www.who.int/ipcs/publications/pesticides_hazard/en/.

[185] C.A. Wilen, Survey of residential pesticide use in the Chollas Creek area of San Diego county and Delhi channel of Orange county, California, California Department of Pesticide Regulation, University of California Statewide IPM Project (UC-IPM), September 2 2002, www.cdpr.ca.gov/ docs/sw/contracts/chollas_survey.pdf.

[186] E.G. Williamson, S.F. Long, M.J. Kallman, M.C. Wilson, A comparative analysis of the acute toxicity of technical-grade pyrethroid insecticides and their commercial formulations, Ecotoxicol. Environ. Saf. 8 (1) (1989) 27-34.

[187] M.J. Wolansky, C. Gennings, K.M. Crofton, Relative potencies for acute effects of pyrethroids on motor function in rats, Toxicol. Sci. 89 (1) (2006) 271-277.

[188] M.J. Wolansky, C. Gennings, K.M. Crofton, Cumulative risk of pyrethroids: testing additivity of the acute motor effects of eleven pyrethroids, Toxicologist 90 (1) (2006) P1203.

[189] M.J. Wolansky, K.L. McDaniel, V.C. Moser, K.M. Crofton, Influence of dosing volume on the neurotoxicity of bifenthrin, Neurotoxicol. Teratol. 29 (3) (2007) 377-384.

[190] M.J. Wolansky, C.M. Mack, P.B. Becker, C.J. Gordon, K.M. Crofton, Effects of acute pyrethroid exposure on thermoregulation in rats, Toxicologist 91 (1) (2007) P423.

[191] C.D.P. Wright, P.J. Forshaw, D.E. Ray, Classification of the actions of ten pyrethroids insecticides in the rat, using the trigeminal reflex and skeletal muscle as test systems, Pestic. Biochem. Physiol. 30 (1988) 79-86.

[192] Z.H. Yang, J.W. Du, Effects of sublethal deltamethrin on the chemical communication system and PBAN activity of the Asian corn borer, $O_{s-}$ trinia furnacalis (Guenee), J. Chem. Ecol. 29 (7) (2003) 1611-1619.

[193] Z.H. Yang, M. Mishimura, K. Nishimura, S. Kuroda, T. Fujita, Quantitative structure-activity studies of pyrethroids. Ch.12: physicochemical substituent effects of meta-phenoxybenzyl disubstituted acetates on insecticidal activity, Pestic. Biochem. Physiol. 29 (1987) 217-232.

[194] E.N. Zerba, Susceptibility and resistance to insecticides of Chagas disease vectors, Medicina (B. Aires) 59 (Suppl. II) (1999) 41-46.

[195] T. Zlotkin, The insect voltage-gated sodium channel as target of insecticides, Annu. Rev. Entomol. 44 (1999) 429-455.

[196] Zucker, Light-dark rhythms in rat eating and drinking behavior, Physiol. Behav. 6 (1971) 115-126. 